\title{
Theory of inelastic lifetimes of low-energy electrons in metals
}

\author{
P.M. Echenique ${ }^{\mathrm{a}, 1}$, J.M. Pitarke ${ }^{\mathrm{b}, 1}$, E.V. Chulkov ${ }^{\mathrm{a}, 1}$, A. Rubio ${ }^{\mathrm{c}}$ \\ ${ }^{a}$ Materialen Fisika Saila, Kimika Fakultatea, Euskal Herriko Unibertsitatea, 1072 Posta kutxatila, 20080 Donostia, Basque Country, Spain \\ ${ }^{\mathrm{b}}$ Materia Kondentsatuaren Fisika Saila, Zientzi Fakultatea, Euskal Herriko Unibertsitatea, 644 Posta kutxatila, 48080 Bilbo, Basque \\ Country, Spain \\ ${ }^{c}$ Departamento de Física Teórica, Universidad de Valladolid, Valladolid 47011, Spain
}

Received 4 March 1999

\begin{abstract}
Electron dynamics in the bulk and at the surface of solid materials are well known to play a key role in a variety of physical and chemical phenomena. In this article we describe the main aspects of the interaction of low-energy electrons with solids, and report extensive calculations of inelastic lifetimes of both low-energy electrons in bulk materials and image-potential states at metal surfaces. New calculations of inelastic lifetimes in a homogeneous electron gas are presented, by using various well-known representations of the electronic response of the medium. Band-structure calculations, which have been recently carried out by the authors and collaborators, are reviewed, and future work is addressed. (C) 2000 Elsevier Science B.V. All rights reserved.
\end{abstract}

PACS: 71.45.Gm; 72.30.+ q; 78.20.-e; 78.70.Ck

\section{Introduction}

Over the years, electron scattering processes in the bulk and at the surface of solid materials have been the subject of a great variety of experimental and theoretical investigations $[1-3]^{2}$. Electron inelastic mean free paths (IMFP) and attenuation lengths have been shown to play a key role in photoelectron spectroscopy and quantitative surface analysis [4-6]. Linewidths of bulk excited electron states in metals have also been measured, with the

\footnotetext{
${ }^{1}$ Donostia International Physics Center (DIPC) and Centro Mixto CSIC-UPV/EHU, Basque Country, Spain.

${ }^{2}$ The factor $\left(1-\gamma^{2} / 3\right)$ in the numerator of $l_{e o}$ of Eq. (2) of Ref. [1] must be replaced by a factor $\sqrt{1-\gamma^{2} / 3}$.
}

use of photoelectron spectroscopy [7-12]. More recently, with the advent of time-resolved two-photon photoemission (TR-2PPE) $[13,14]$ and ultrafast laser technology, time domain measurements of the lifetimes of photoexcited electrons with energies below the vacuum level have been performed. In these experiments, the lifetimes of both hot electrons in bulk materials [15-29] and image-potential states at metal surfaces [29-35] have been probed.

These new and powerful experimental techniques, based on high resolution direct and inverse photoemission as well as time-resolved measurements, have addressed aspects related to the lifetime of excited electrons and have raised many fundamental questions. The ultrafast laser technology has allowed to probe fast events at surfaces in real time and, therefore, extract information about elementary electronic 
processes (with time scales from pico to femtoseconds) that are relevant for potential technological applications. In general, the two-photon photoemission spectroscopy is sensitive to changes of geometries, local work functions, and surface potentials during layer formation. The interaction of excited electrons and the underlying substrate governs the cross-section and branching ratios of all electronically induced adsorbate reactions at surfaces, such as dissociation or desorption, and influences the reactivity of the surfaces as well as the kinetics of growth [36]. Hot-electron lifetimes have long been invoked to give valuable information about these processes.

Inelastic lifetimes of excited electrons with energies larger than $\sim 1 \mathrm{eV}$ above the Fermi level can be attributed to electron-electron (e-e) inelastic scattering, other processes such as electron-phonon and electron-imperfection interactions being, in general, of minor importance ${ }^{3}$. A self-consistent calculation of the interaction of low-energy electrons with an electron gas was first carried out by Quinn and Ferrell [38]. They performed a self-energy calculation of e-e scattering rates near the Fermi surface, and derived a formula for the inelastic lifetime of hot electrons that is exact in the high-density limit. These free-electron-gas (FEG) calculations were extended by Ritchie [39] ${ }^{4}$ and Quinn [41] to include, within the first-Born and random-phase approximations, energies away from the Fermi surface, and by Adler [42] and Quinn [43] to take account of the effects of the presence of a periodic lattice and, in particular, the effect of virtual interband transitions [43]. Since then, several FEG calculations of e-e scattering rates have been performed, with inclusion of exchange and correlation (XC) effects [44-47], chemical potential renormalization $[48,49]$, plasmon damping [50], and core polarizability [51]. In the case of free-electron materials, such as aluminum, valence electrons were described within the FEG model and atomic generalized oscillator strengths were used for inner-shell ionization [51,52]. For the description of the IMFP in non-free-electron metals,

\footnotetext{
${ }^{3}$ Electron relaxation times due to coupling with the lattice are found to be on a picosecond scale (see, e.g., Ref. [37]).

${ }^{4}$ The $1 / 2$ factor in front of $z^{2}$ in the expansion of $f_{1}$ just before Eq. (6.15) of this reference must be replaced by $1 / 3$, as done in a subsequent paper, [40].
}

Krolikowski and Spicer [53] employed a semiempirical approach to calculate the energy dependence of the IMFP from the knowledge of density-of-state distributions, which had been deduced from photoelectron energy-distribution measurements. Tung et al. [54], used a statistical approximation, assuming that the inelastic scattering of an electron in a given volume element of the solid can be represented by the scattering appropriate to a FEG with the electron density in that volume element. This approximation was found to predict IMFPs for electrons in $\mathrm{Al}$ that are in good agreement with predictions from an electron gas model plus atomic inner-shell contributions, and these authors [54] went further to evaluate IMFPs and energy losses in various noble and transition metals. Later on, new methods were proposed [55-59] for calculating the IMFP, which were based on a model dielectric function whose form was motivated by the use of optical data. Though highenergy electron mean free paths now seem to be well understood [60-62], in the low-energy domain electrons are more sensitive to the details of the band structure of the solid, and a treatment of the electron dynamics that fully includes band structure effects is necessary for quantitative comparisons with experimentally determined attenuation lengths and relaxation times. $\mathrm{Ab}$ initio calculations of these quantities in which both the electronic Bloch states of the probe electron and the dielectric response function of the medium are described from first principles have been performed only very recently $[63,64]$.

The self-energy formalism first introduced by Quinn and Ferrell for the description of the lifetime of hot electrons in a homogeneous electron gas was extended by Echenique et al. [65-67] to quantitatively evaluate the lifetime of image-potential states [68-75] at metal surfaces. Echenique et al. [65-67] used hydrogenic-like states to describe the imagestate wave functions, they introduced a step model potential to calculate the bulk final-state wave functions, and used simplified free-electron-gas models to approximate the screened Coulomb interaction. A three band model was used by Gao and Lundqvist [76] to describe the band structure of the (111) surfaces of copper and nickel. They calculated, in terms of Auger transitions, the decay of the first image state on these surfaces to the $n=0$ crystal-induced surface state, neglecting screening effects. 
Self-consistent calculations of the linewidths of image states on copper surfaces have been reported recently [77-80], and good agreement with experimentally determined decay times has been found. These calculations were performed by going beyond a free-electron description of the metal surface. Single-particle wave functions were obtained by solving the Schrödinger equation with a realistic one-dimensional model potential [81], and the screened interaction was evaluated in the random-phase approximation (RPA).

This paper includes an overview of inelastic lifetimes of low-energy electrons in the bulk and at the surface of solid materials, as derived within the first-Born approximation or, equivalently, linear response theory. In the framework of linear response theory, the inelastic energy broadening or lifetimewidth of probe particles interacting with matter is found to be proportional to the square of the probe charge. Extensions that include the quadratic response to external perturbations have been discussed by various authors [82-88], in order to give account of the existing dependence of the energy loss and the IMFP on the sign of the projectile charge $[89,90]$.

Section 2 is devoted to the study of electron scattering processes in a homogeneous electron gas, employing various representations of the electronic response of the medium. In Section 3, a general self-energy formulation appropriate for the description of inhomogeneous many-body systems is introduced. This formulation is applied in Sections 4 and 5 to review theoretical investigations of lifetimes of both hot electrons in bulk materials and image-potential states at metal surfaces. Future work is addressed in Section 6.

Unless otherwise is stated, atomic units are used throughout, i.e., $e^{2}=\hbar=m_{e}=1$. The atomic unit of length is the Bohr radius, $a_{0}=\hbar^{2} / m_{e}^{2}=0.529 \AA$, the atomic unit of energy is the Hartree, 1 Hartree $=$ $e^{2} / a_{0}=27.2 \mathrm{eV}$, and the atomic unit of velocity is the Bohr velocity, $v_{0}=\alpha c=2.19 \times 10^{8} \mathrm{~cm} \mathrm{~s}^{-1}, \alpha$ and $c$ being the fine structure constant and the velocity of light, respectively.

\section{Scattering theory approach}

We take a homogeneous system of interacting electrons, and consider an excited electron interact-

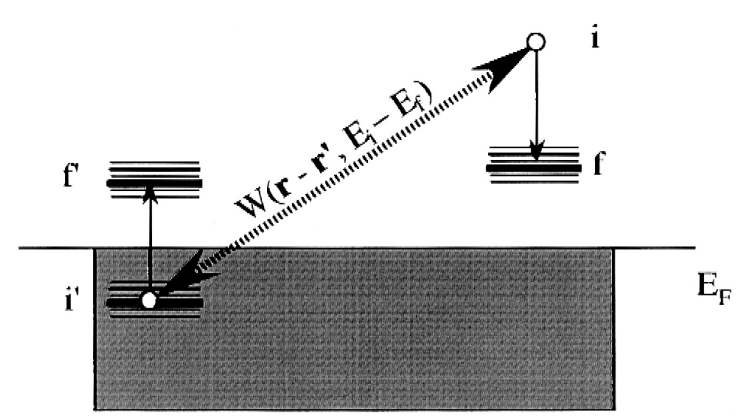

Fig. 1. Scattering of an excited electron with the Fermi sea. The probe electron is scattered from a state $\phi_{i}(\boldsymbol{r})$ of energy $E_{i}$ to some other state $\phi_{f}(\boldsymbol{r})$ of energy $E_{f}$, by carrying one electron of the Fermi sea from an initial state $\phi_{i^{\prime}}(\boldsymbol{r})$ of energy $E_{i^{\prime}}$ to a final state $\phi_{f^{\prime}}(\boldsymbol{r})$ of energy $E_{f^{\prime}}$, according to a dynamic screened interaction $W\left(\boldsymbol{r}-\boldsymbol{r}^{\prime}, E_{i}-E_{f}\right) . E_{\mathrm{F}}$ represents the Fermi level.

ing through individual collisions with electrons in the Fermi sea. Hence, we calculate the probability $P_{i, i^{\prime}}^{f, f^{\prime}}$ per unit time corresponding to the process by which the probe particle is scattered from a state $\phi_{i}(\boldsymbol{r})$ of energy $E_{i}$ to some other state $\phi_{f}(\boldsymbol{r})$ of energy $E_{f}$, by carrying one electron of the Fermi sea from an initial state $\phi_{i^{\prime}}(\boldsymbol{r})$ of energy $E_{i^{\prime}}$ to a final state $\phi_{f^{\prime}}(\boldsymbol{r})$ of energy $E_{f^{\prime}}$, according to a dynamic screened interaction $W\left(\boldsymbol{r}-\boldsymbol{r}^{\prime} ; E_{i}-E_{f}\right)$ (see Fig. 1). By using the 'golden rule' of time-dependent perturbation theory and keeping only terms of first order in the screened interaction, one writes [91]:

$$
\begin{aligned}
P_{i, i^{\prime}}^{f, f^{\prime}}= & 2 \pi\left|\left[W\left(\boldsymbol{r}-\boldsymbol{r}^{\prime} ; E_{i}-E_{f}\right)\right]_{i, i^{\prime}}^{f, f^{\prime}}\right|^{2} \\
& \times \delta\left(E_{i}-E_{f}+E_{i}^{\prime}-E_{f}^{\prime}\right),
\end{aligned}
$$

where

$$
\begin{aligned}
{\left[W\left(\boldsymbol{r}-\boldsymbol{r}^{\prime} ; \omega\right)\right]_{i, i^{\prime}}^{f, f^{\prime}}=} & \int \mathrm{d} \boldsymbol{r} \int \mathrm{d} \boldsymbol{r}^{\prime} \phi_{i}^{*}(\boldsymbol{r}) \phi_{i^{\prime}}^{*}\left(\boldsymbol{r}^{\prime}\right) \\
& \times W\left(\boldsymbol{r}-\boldsymbol{r}^{\prime} ; \omega\right) \phi_{f}(\boldsymbol{r}) \phi_{f^{\prime}}\left(\boldsymbol{r}^{\prime}\right) .
\end{aligned}
$$

Using plane waves for all initial and final states,

$$
\phi_{\boldsymbol{k}}(\boldsymbol{r})=\frac{1}{\sqrt{\Omega}} \mathrm{e}^{\mathrm{i} \boldsymbol{k} \cdot \boldsymbol{r}},
$$


with energy $\omega_{k}=k^{2} / 2$ and $\Omega$ being the normalization volume, one finds

$$
\begin{aligned}
P_{i, i^{\prime}}^{f, f^{\prime}}= & \frac{2 \pi}{\Omega^{2}}\left|W_{\boldsymbol{k}_{i}-\boldsymbol{k}_{f}, \omega_{\boldsymbol{k}_{i}}-\omega_{\boldsymbol{k}_{f}}}\right|^{2} \delta_{\boldsymbol{k}_{i}-\boldsymbol{k}_{f}-\boldsymbol{k}_{f}^{\prime}+\boldsymbol{k}_{i}^{\prime}} \\
& \times \delta\left(\omega_{\boldsymbol{k}_{i}}-\omega_{\boldsymbol{k}_{f}}-\omega_{\boldsymbol{k}_{f}^{\prime}}+\omega_{\boldsymbol{k}_{i}^{\prime}}\right),
\end{aligned}
$$

where $W_{q, \omega}$ represents the Fourier transform of the screened interaction $W\left(\boldsymbol{r}-\boldsymbol{r}^{\prime} ; \omega\right)$. The Kroenecker delta and the Dirac delta function on the right-hand side of Eq. (4) allow for wave-vector and energy conservation, respectively.

By summing the probabilities $P_{i, i^{\prime}}^{f, f^{\prime}}$ of Eq. (4) over all possible states $\boldsymbol{k}_{i}^{\prime}\left(k_{i}^{\prime}<q_{\mathrm{F}}, q_{\mathrm{F}}\right.$ being the Fermi momentum), $\boldsymbol{k}_{f}^{\prime}\left(k_{f}^{\prime}>q_{\mathrm{F}}\right)$ and $\boldsymbol{k}_{f}$, and noting that each allowed $\boldsymbol{k}_{i}^{\prime}$ leads to two one-electron states (one for each spin), the total scattering rate of the probe electron in the state $\boldsymbol{k}_{i}$ is found to be given by the following expression:

$$
\begin{aligned}
\tau^{-1}= & \frac{4 \pi}{\Omega^{2}} \sum_{\boldsymbol{q}}^{\prime} \sum_{\boldsymbol{k}_{i}^{\prime}}\left|W_{\boldsymbol{q}, \omega}\right|^{2} n_{\boldsymbol{k}_{i}^{\prime}}\left(1-n_{\boldsymbol{k}_{i}^{\prime}+\boldsymbol{q}}\right) \\
& \times \delta\left(\omega-\omega_{\boldsymbol{k}_{i}^{\prime}+\boldsymbol{q}}+\omega_{\boldsymbol{k}_{i}^{\prime}}\right),
\end{aligned}
$$

where

$n_{k}=\theta\left(q_{\mathrm{F}}-|\boldsymbol{k}|\right)$

represents the occupation number. We have set the energy transfer $\omega=\omega_{k_{i}}-\omega_{k_{i}-q}$, and the prime in the summation indicates that the momentum transfer is subject to the condition $0<\omega<\omega_{\boldsymbol{k}_{i}}-E_{\mathrm{F}}\left(E_{\mathrm{F}}\right.$ is the Fermi energy), accounting for the fact that the probe electron cannot make transitions to occupied states in the Fermi sea.

With the interaction $W_{q, \omega}$ described by the bare Coulomb interaction, that is, $W_{\boldsymbol{q}, \omega}=v_{q}$, the summation over $\boldsymbol{q}$ in Eq. (5) would be severely divergent, thereby resulting in an infinite damping rate. Instead, we assume that the Coulomb interaction is dynamically screened,

$W_{q, \omega}=\epsilon_{q, \omega}^{-1} v_{q}$,

where $\epsilon_{q, \omega}$ is taken to be the dielectric function of the medium $[92,93]$.

For $\omega>0$, the imaginary part of the RPA dielectric function [94,95] is simply a measure of the number of states available for real transitions involv- ing a given momentum transfer $\boldsymbol{q}$ and energy transfer $\omega$ :

$$
\begin{aligned}
\operatorname{Im} \epsilon_{q, \omega}^{\mathrm{RPA}}= & 2 \pi \Omega^{-1} v_{q} \sum_{k} n_{k}\left(1-n_{k+q}\right) \\
& \times \delta\left(\omega-\omega_{k+q}+\omega_{k}\right) .
\end{aligned}
$$

In the limit that the volume of the system $\Omega$ becomes infinite, one can replace sums over states by integrals with the following relation

$$
\sum_{\boldsymbol{k}} f(\boldsymbol{k}) \rightarrow \frac{\Omega}{(2 \pi)^{3}} \int \mathrm{d} \boldsymbol{k} f(\boldsymbol{k}),
$$

and after introduction of Eq. (8) into Eq. (5), one finds

$$
\tau^{-1}=2 \int^{\prime} \frac{\mathrm{d} \boldsymbol{q}}{(2 \pi)^{3}} v_{\boldsymbol{q}} \frac{\operatorname{Im} \epsilon_{\boldsymbol{q}, \omega}^{\mathrm{RPA}}}{\left|\epsilon_{\boldsymbol{q}, \omega}\right|^{2}},
$$

where the prime in the integration indicates that the momentum transfer $\boldsymbol{q}$ is subject to the same condition as in Eq. (5). With the screened interaction $W_{q, \omega}$ of Eq. (7) described within RPA, one writes

$\tau^{-1}=2 \int^{\prime} \frac{\mathrm{d} \boldsymbol{q}}{(2 \pi)^{3}} v_{\boldsymbol{q}} \operatorname{Im}\left[-\boldsymbol{\epsilon}_{\boldsymbol{q}, \omega}^{-1}\right]$,

with $\epsilon_{q, \omega}$ being the RPA dielectric function [94,95], i.e., $\epsilon_{q, \omega}=\epsilon_{q, \omega}^{\mathrm{RPA}}$.

In the more general scenario of many-body theory and within the first Born approximation [96], one finds the damping rate of an excited electron in the state $\boldsymbol{k}_{i}$ to also be given by Eq. (11), but with the exact inverse dielectric function $\epsilon_{q, \omega}^{-1}$, as defined in Appendix A. This is the result obtained independently by Quinn and Ferrell [38] and by Ritchie [39]. Quinn and Ferrell [38] demonstrated, within a selfenergy formalism, that the damping rate of holes below the Fermi level is also given by Eq. (11), with the energy transfer $\omega=\omega_{\boldsymbol{k}_{i}-q}-\omega_{\boldsymbol{k}_{i}}$ and with the prime in the integration indicating that the momentum transfer $\boldsymbol{q}$ is subject to the condition $0<\omega<E_{\mathrm{F}}$ $-\omega_{\boldsymbol{k}_{i}}$. For small values of $\left|\omega_{\boldsymbol{k}_{i}}-E_{\mathrm{F}}\right|$, holes inside the Fermi sea $\left(\omega_{\boldsymbol{k}_{i}}<E_{\mathrm{F}}\right)$ are found to damp out in the same way as electrons outside $\left(\omega_{\boldsymbol{k}_{i}}>E_{\mathrm{F}}\right)$, as shown in Fig. 2.

If one is to go beyond RPA and introduce, through the factor $\left(1-G_{q, \omega}\right)$ (see Appendix A), the reduction in the e-e interaction due to the existence of a 


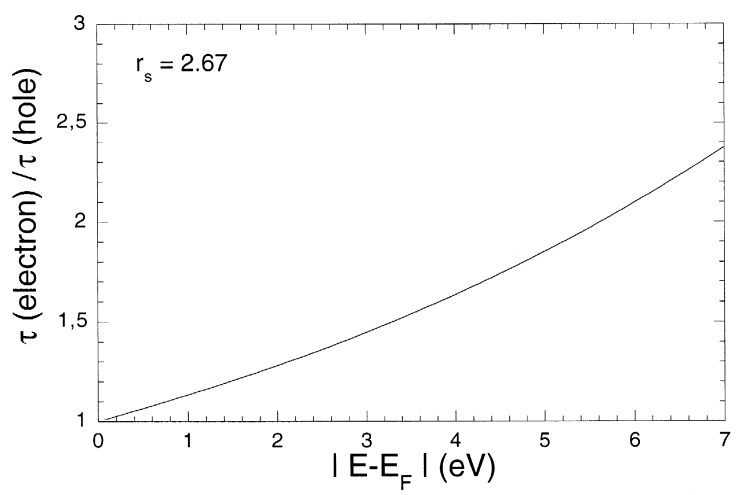

Fig. 2. Ratio of the lifetime of electrons above the Fermi level $\left(E>E_{\mathrm{F}}\right)$ to the lifetime of holes below the Fermi level $\left(E<E_{\mathrm{F}}\right)$, as a function of $\left|E-E_{\mathrm{F}}\right|$, calculated within RPA for an electron density equal to that of valence $\left(4 s^{1}\right)$ electrons in copper, i.e., $r_{s}=2.67$.

local XC hole around electrons in the Fermi sea, the dielectric function entering Eq. (11) is

$\epsilon_{q, \omega}=1+\frac{\epsilon_{q, \omega}^{\mathrm{RPA}}-1}{1-G_{q, \omega}\left(\epsilon_{q, \omega}^{\mathrm{RPA}}-1\right)}$,

where $G_{q, \omega}$ is the so-called local-field factor, first introduced by Hubbard [97].

If one accounts, through the factor $\left(1-G_{\boldsymbol{q}, \omega}\right)$, for the existence of a local XC hole around electrons in the Fermi sea and also around the probe electron, the dielectric function entering Eq. (11) is the so-called test-charge-electron dielectric function [98,99] (see Appendix A):

$\epsilon_{q, \omega}=\epsilon_{q, \omega}^{\mathrm{RPA}}-G_{q, \omega}\left(\epsilon_{q, \omega}^{\mathrm{RPA}}-1\right)$.

Finally, we note that the inelastic mean free path (IMFP) is directly connected to the lifetime $\tau$ through the relation

$\lambda=v \tau$.

\section{Self-energy formalism}

In the framework of many-body theory [96], the damping rate of an electron with energy $\varepsilon_{i}>E_{\mathrm{F}}$ is obtained from the imaginary part of the electron self-energy:

$$
\tau^{-1}=-2 \int \mathrm{d} \boldsymbol{r} \int \mathrm{d} \boldsymbol{r}^{\prime} \phi_{i}^{*}(\boldsymbol{r}) \operatorname{Im} \Sigma\left(\boldsymbol{r}, \boldsymbol{r}^{\prime} ; \varepsilon_{i}\right) \phi_{i}\left(\boldsymbol{r}^{\prime}\right),
$$

where $\phi_{i}(\boldsymbol{r})$ represents a suitably chosen one-electron orbital of energy $\varepsilon_{i}$ (see Appendix B).

In the GW approximation [100,101], one considers only the first-order term in a series expansion of the self-energy in terms of the screened interaction $W\left(\boldsymbol{r}, \boldsymbol{r}^{\prime}, \omega\right)$. This is related to the density-response function $\chi\left(\boldsymbol{r}, \boldsymbol{r}^{\prime}, \omega\right)$ of Eq. (A.2), as follows

$$
\begin{aligned}
W\left(\boldsymbol{r}, \boldsymbol{r}^{\prime} ; \omega\right)= & v\left(\boldsymbol{r}-\boldsymbol{r}^{\prime}\right)+\int \mathrm{d} \boldsymbol{r}_{1} \int \mathrm{d} \boldsymbol{r}_{2} v\left(\boldsymbol{r}-\boldsymbol{r}_{1}\right) \\
& \times \chi\left(\boldsymbol{r}_{1}, \boldsymbol{r}_{2}, \omega\right) v\left(\boldsymbol{r}_{2}-\boldsymbol{r}^{\prime}\right),
\end{aligned}
$$

where $v\left(\boldsymbol{r}-\boldsymbol{r}^{\prime}\right)$ represents the bare Coulomb potential.

Within RPA, the density-response function satisfies and integral equation (see Eq. (A.6)), and is obtained from the knowledge of the density-response function of noninteracting electrons. If, to the same order of approximation, one replaces the exact oneparticle Green function by its noninteracting counterpart, the imaginary part of the self-energy can be evaluated explicitly:

$$
\begin{aligned}
\operatorname{Im} & \sum\left(\boldsymbol{r}, \boldsymbol{r}^{\prime} ; \varepsilon_{i}>E_{\mathrm{F}}\right) \\
= & \sum_{f}^{\prime} \phi_{f}^{*}\left(\boldsymbol{r}^{\prime}\right) \operatorname{Im} W\left(\boldsymbol{r}, \boldsymbol{r}^{\prime} ; \omega\right) \phi_{f}(\boldsymbol{r}),
\end{aligned}
$$

where $\omega=\varepsilon_{i}-\varepsilon_{f}$, and the prime in the summation indicates that states $\phi_{f}(\boldsymbol{r})$ available for real transitions are subject to the condition that $0<\omega<\varepsilon_{i}-$ $E_{\mathrm{F}}$. Introduction of Eq. (17) into Eq. (15) yields

$$
\begin{aligned}
\tau^{-1}= & -2 \sum_{f}^{\prime} \int \mathrm{d} \boldsymbol{r} \int \mathrm{d} \boldsymbol{r}^{\prime} \phi_{i}{ }^{*}(\boldsymbol{r}) \phi_{f}^{*}\left(\boldsymbol{r}^{\prime}\right) \\
& \times \operatorname{Im} W\left(\boldsymbol{r}, \boldsymbol{r}^{\prime} ; \omega\right) \phi_{i}\left(\boldsymbol{r}^{\prime}\right) \phi_{f}^{*}(\boldsymbol{r}) .
\end{aligned}
$$

In the so-called GW $\Gamma$ approximation [102-105], which includes XC effects not present in the GWRPA, the self-energy and damping rate of the excited electron are of the $\mathrm{GW}$ form, i.e., they are given by 
Eqs. (17) and (18), respectively, but with an effective screened interaction

$$
\begin{aligned}
& W\left(\boldsymbol{r}, \boldsymbol{r}^{\prime} ; \omega\right) \\
& =v\left(\boldsymbol{r}-\boldsymbol{r}^{\prime}\right)+\int \mathrm{d} \boldsymbol{r}_{1} \int \mathrm{d} \boldsymbol{r}_{2}\left[v\left(\boldsymbol{r}-\boldsymbol{r}_{1}\right)\right. \\
& \left.\quad+K^{\mathrm{xc}}\left(\boldsymbol{r}, \boldsymbol{r}_{1}\right)\right] \chi\left(\boldsymbol{r}_{1}, \boldsymbol{r}_{2}, \omega\right) v\left(\boldsymbol{r}_{2}-\boldsymbol{r}^{\prime}\right),
\end{aligned}
$$

the density-response function now being given by Eq. (A.8). The kernel $K^{\mathrm{xc}}\left(\boldsymbol{r}, \boldsymbol{r}^{\prime}\right)$ entering Eqs. (19) and (A.8) accounts for the reduction in the e-e interaction due to the existence of short-range $\mathrm{XC}$ effects associated to the probe electron and to screening electrons, respectively.

\subsection{Homogeneous electron gas}

In the case of a homogeneous electron gas, single-particle wave functions are simply plane waves, as defined in Eq. (3). By introducing these orbitals into Eq. (18), the damping rate of an electron in the state $\boldsymbol{k}_{i}$ is found to be given by Eq. (11) with the dielectric function of either Eq. (12) or Eq. (13), depending on weather the screened interaction of Eq. (16) or Eq. (19) is taken in combination with the density-response function of Eq. (A.8) ${ }^{5}$. This is an expected result, since these calculations have all been performed to lowest order in the screened interaction.

\subsection{Bounded electron gas}

In the case of a bounded electron gas that is translationally invariant in the plane of the surface, single-particle wave functions are of the form

$\phi_{\boldsymbol{k}_{\|}, i}(\boldsymbol{r})=\frac{1}{\sqrt{A}} \phi_{i}(z) \mathrm{e}^{\mathrm{i} \boldsymbol{k}_{\|} \cdot \boldsymbol{r} \|}$,

with energies

$\varepsilon_{\boldsymbol{k}_{\|}, i}=\varepsilon_{i}+\frac{\boldsymbol{k}_{\|}^{2}}{2}$,

where the $z$-axis has been taken to be perpendicular to the surface. Hence, the wave functions $\phi_{i}(z)$ and

\footnotetext{
${ }^{5}$ If Eq. (16) for the screened interaction is taken in combination with the RPA density-response function of Eq. (A.6), then one obtains Eq. (11) with the RPA dielectric function.
}

energies $\varepsilon_{i}$ describe motion normal to the surface, $\boldsymbol{k}_{\|}$is a wave vector parallel to the surface, and $A$ is the normalization area.

Introduction of Eq. (20) into Eqs. (15) and (18) yields the following expressions for the damping rate of an electron in the state $\phi_{\boldsymbol{k}_{\|}, i}(\boldsymbol{r})$ with energy $\boldsymbol{\varepsilon}_{\boldsymbol{k}_{\|}, i}$ :

$$
\begin{aligned}
\tau^{-1}= & -2 \int \mathrm{d} z \int \mathrm{d} z^{\prime} \int \frac{\mathrm{d} \boldsymbol{q}_{\|}}{(2 \pi)^{2}} \phi_{i}{ }^{*}(z) \\
& \times \operatorname{Im} \Sigma\left(z, z^{\prime} ; \boldsymbol{q}_{\|}, \varepsilon_{\boldsymbol{k}_{\|}, i}\right) \phi_{i}\left(z^{\prime}\right)
\end{aligned}
$$

and

$$
\begin{aligned}
\tau^{-1}= & -2 \sum_{f}^{\prime} \int \mathrm{d} z \int \mathrm{d} z^{\prime} \int \frac{\mathrm{d} \boldsymbol{q}_{\|}}{(2 \pi)^{2}} \phi_{i}{ }^{*}(z) \phi_{f}^{*}\left(z^{\prime}\right) \\
& \times \operatorname{Im} W\left(z, z^{\prime} ; \boldsymbol{q}_{\|}, \omega\right) \phi_{f}(z) \phi_{i}\left(z^{\prime}\right),
\end{aligned}
$$

respectively, where $\omega=\varepsilon_{\boldsymbol{k}_{\|}, i}-\varepsilon_{\boldsymbol{k}_{\|}-\boldsymbol{q}_{\|}, f}$. Here, $\Sigma\left(z, z^{\prime} ; \boldsymbol{q}_{\|}, \omega\right)$ and $W\left(z, z^{\prime} ; \boldsymbol{q}_{\|}, \omega\right)$ represent the twodimensional Fourier transforms of the electron selfenergy $\Sigma\left(\boldsymbol{r}, \boldsymbol{r}^{\prime} ; \omega\right)$ and the screened interaction $W\left(\boldsymbol{r}, \boldsymbol{r}^{\prime} ; \omega\right)$.

\subsection{Periodic crystals}

For periodic crystals, single-particle wave functions are Bloch states

$\phi_{\boldsymbol{k}, i}(\boldsymbol{r})=\frac{1}{\sqrt{\Omega}} \mathrm{e}^{\mathrm{i} \boldsymbol{k} \cdot \boldsymbol{r}} u_{\boldsymbol{k}, i}(\boldsymbol{r})$,

and one may introduce the following Fourier expansion of the screened interaction:

$$
\begin{aligned}
W\left(\boldsymbol{r}, \boldsymbol{r}^{\prime} ; \omega\right)= & \int_{\mathrm{BZ}} \frac{\mathrm{d} \boldsymbol{q}}{(2 \pi)^{3}} \sum_{\boldsymbol{G}} \sum_{\boldsymbol{G}^{\prime}} \mathrm{e}^{\mathrm{i}(\boldsymbol{q}+\boldsymbol{G}) \cdot \boldsymbol{r}} \mathrm{e}^{-\mathrm{i}\left(\boldsymbol{q}+\boldsymbol{G}^{\prime}\right) \cdot \boldsymbol{r}^{\prime}} \\
& \times W_{\boldsymbol{G}, \boldsymbol{G}^{\prime}}(\boldsymbol{q}, \omega),
\end{aligned}
$$

where the integration over $\boldsymbol{q}$ is extended over the first Brillouin zone (BZ), and the vectors $\boldsymbol{G}$ and $\boldsymbol{G}^{\prime}$ are reciprocal lattice vectors. Introducing this Fourier representation into Eq. (18), one finds the following expression for the damping rate of an electron in the state $\phi_{k, i}(\boldsymbol{r})$ with energy $\varepsilon_{\boldsymbol{k}, i}$ :

$$
\begin{aligned}
\tau^{-1}= & -2 \sum_{f}^{\prime} \int_{\mathrm{BZ}} \frac{\mathrm{d} \boldsymbol{q}}{(2 \pi)^{3}} \sum_{\boldsymbol{G}} \sum_{\boldsymbol{G}^{\prime}} B_{i f}^{*}(\boldsymbol{q}+\boldsymbol{G}) \\
& \times B_{i f}\left(\boldsymbol{q}+\boldsymbol{G}^{\prime}\right) \operatorname{Im} W_{\boldsymbol{G}, \boldsymbol{G}^{\prime}}(\boldsymbol{q}, \omega),
\end{aligned}
$$


or, equivalently,

$$
\begin{aligned}
& \tau^{-1}=\frac{1}{\pi^{2}} \sum_{f}^{\prime} \int_{\mathrm{BZ}} \mathrm{d} \boldsymbol{q} \sum_{\boldsymbol{G}} \sum_{\boldsymbol{G}^{\prime}} \frac{B_{i f}^{*}(\boldsymbol{q}+\boldsymbol{G}) B_{i f}\left(\boldsymbol{q}+\boldsymbol{G}^{\prime}\right)}{|\boldsymbol{q}+\boldsymbol{G}|^{2}} \\
& \qquad \times \operatorname{Im}\left[-\boldsymbol{\epsilon}_{\boldsymbol{G}, \boldsymbol{G}^{\prime}}^{-1}(\boldsymbol{q}, \omega)\right], \\
& \text { where } \omega=\varepsilon_{\boldsymbol{k}, i}-\varepsilon_{\boldsymbol{k}-\boldsymbol{q}, f}, \text { and } \\
& B_{i f}(\boldsymbol{q}+\boldsymbol{G})=\int \mathrm{d} \boldsymbol{r} \phi_{\boldsymbol{k}, i}^{*}(\boldsymbol{r}) \mathrm{e}^{\mathrm{i}(\boldsymbol{q}+\boldsymbol{G}) \cdot \boldsymbol{r}} \phi_{\boldsymbol{k}-\boldsymbol{q}, f}(\boldsymbol{r}) .
\end{aligned}
$$

$W_{G, G^{\prime}}(\boldsymbol{q}, \omega)$ are the Fourier coefficients of the screened interaction, and $\epsilon_{G, G^{\prime}}^{-1}(\boldsymbol{q}, \omega)$ are the Fourier coefficients of the inverse dielectric function.

Within RPA, one writes

$\boldsymbol{\epsilon}_{\boldsymbol{G}, \boldsymbol{G}^{\prime}}(\boldsymbol{q}, \omega)=\delta_{\boldsymbol{G}, \boldsymbol{G}^{\prime}}-\chi_{\boldsymbol{G}, \boldsymbol{G}^{\prime}}^{0}(\boldsymbol{q}, \omega) v_{\boldsymbol{G}^{\prime}}(\boldsymbol{q})$,

where $v_{G}(\boldsymbol{q})$ represent the Fourier coefficients of the bare Coulomb potential,

$v_{\boldsymbol{G}}(\boldsymbol{q})=\frac{4 \pi}{|\boldsymbol{q}+\boldsymbol{G}|^{2}}$,

and $\chi_{\boldsymbol{G}, \boldsymbol{G}^{\prime}}^{0}(\boldsymbol{q}, \omega)$ are the Fourier coefficients of the density-response function of noninteracting electrons,

$$
\begin{aligned}
\chi_{\boldsymbol{G}, \boldsymbol{G}^{\prime}}^{0}(\boldsymbol{q}, \omega)= & 2 \int_{\mathrm{BZ}} \frac{\mathrm{d} \boldsymbol{k}}{(2 \pi)^{3}} \sum_{n} \sum_{n^{\prime}} \\
& \times \frac{f_{\boldsymbol{k}, n}-f_{\boldsymbol{k}+\boldsymbol{q}, n^{\prime}}}{\varepsilon_{\boldsymbol{k}, n}-\varepsilon_{\boldsymbol{k}+\boldsymbol{q}, n^{\prime}}+(\omega+\mathrm{i} \eta)} \\
& \times\left\langle\phi_{\boldsymbol{k}, n}\left|\mathrm{e}^{-\mathrm{i}(\boldsymbol{q}+\boldsymbol{G}) \cdot \boldsymbol{r}}\right| \phi_{\boldsymbol{k}+\boldsymbol{q}, n^{\prime}}\right\rangle \\
& \times\left\langle\phi_{\boldsymbol{k}+\boldsymbol{q}, n^{\prime}}\left|\mathrm{e}^{\mathrm{i}\left(\boldsymbol{q}+\boldsymbol{G}^{\prime}\right) \cdot \boldsymbol{r}}\right| \phi_{\boldsymbol{k}, n}\right\rangle,
\end{aligned}
$$

$\eta$ being a positive infinitesimal. The sums run over the band structure for each wave wave vector $\boldsymbol{k}$ in the first BZ, and $f_{k, n}$ are Fermi factors

$f_{k, n}=\theta\left(E_{\mathrm{F}}-\varepsilon_{k, n}\right)$.

Couplings of the wave vector $\boldsymbol{q}+\boldsymbol{G}$ to wave vectors $\boldsymbol{q}+\boldsymbol{G}^{\prime}$ with $\boldsymbol{G} \neq \boldsymbol{G}^{\prime}$ appear as a consequence of the existence of electron-density variations in real solids. If these terms, representing the so-called crystalline local-field effects, are neglected, one can write

$$
\begin{aligned}
\tau^{-1}= & \frac{1}{\pi^{2}} \sum_{f}^{\prime} \int_{\mathrm{BZ}} \mathrm{d} \boldsymbol{q} \sum_{\boldsymbol{G}} \frac{\left|B_{i f}(\boldsymbol{q}+\boldsymbol{G})\right|^{2}}{|\boldsymbol{q}+\boldsymbol{G}|^{2}} \\
& \times \frac{\operatorname{Im}\left[\boldsymbol{\epsilon}_{\boldsymbol{G}, \boldsymbol{G}}(\boldsymbol{q}, \omega)\right]}{\left|\epsilon_{\boldsymbol{G}, \boldsymbol{G}}(\boldsymbol{q}, \omega)\right|^{2}}
\end{aligned}
$$

The imaginary part of $\epsilon_{G, G}(q, \omega)$ represents a measure of the number of states available for real transitions involving a given momentum and energy transfer $\boldsymbol{q}+\boldsymbol{G}$ and $\boldsymbol{\omega}$, respectively, and the factor $\left|\boldsymbol{\epsilon}_{\boldsymbol{G}, \boldsymbol{G}}(\boldsymbol{q}, \omega)\right|^{-2}$ accounts for the screening in the interaction with the probe electron. Initial and final states of the probe electron enter through the coefficients $B_{i f}(\boldsymbol{q}+\boldsymbol{G})$.

If one further replaces in Eq. (33) the probe electron initial and final states by plane waves, and the matrix coefficients $\epsilon_{G, G}(\boldsymbol{q}, \omega)$ by the dielectric function of a homogeneous electron gas,

$\epsilon_{G, G}(q, \omega) \rightarrow \epsilon(|q+G|, \omega)$,

then Eq. (33) yields the damping rate of excited electrons in a FEG, as given by Eq. (11).

We note that the hot-electron decay in real solids depends on both the wave vector $\boldsymbol{k}$ and the band index $i$ of the initial Bloch state. As a result of the symmetry of these states, one finds that $\tau^{-1}(S k, i)=$ $\tau^{-1}(\boldsymbol{k}, i)$, with $S$ representing a point group symmetry operation in the periodic crystal. Hence, for each value of the hot-electron energy the scattering rate $\tau^{-1}(E)$ is defined by averaging $\tau^{-1}(\boldsymbol{k}, i)$ over all wave vectors $\boldsymbol{k}$ lying in the irreducible element of the Brillouin zone (IBZ), with the same energy, and also over the band structure for each wave vector.

\section{Lifetimes of hot electrons in metals}

\subsection{Jellium model}

Early calculations of inelastic lifetimes and mean free paths of excited electrons in metals were based on the 'jellium' model of the solid. Within this model, valence electrons are described by a homogeneous assembly of electrons immersed in a uniform background of positive charge and volume $\Omega$. The only parameter in this model is the valence-electron density $n_{0}$, which we represent in terms of the so-called electron-density parameter $r_{s}$ defined by the relation $1 / n_{0}=(4 / 3) \pi\left(r_{s} a_{0}\right)^{3}, a_{0}$ being the Bohr radius. Hence, the damping rate of a hot electron of energy $E=\omega_{\boldsymbol{k}_{i}}$ is obtained, within this model, from Eq. (11) with $\omega=\omega_{\boldsymbol{k}_{i}}-\omega_{\boldsymbol{k}_{i}-q}$. 
In the high-density limit $\left(r_{s} \rightarrow 0\right), \mathrm{XC}$ effects as well as high-order terms in the expansion of the scattering probability in terms of the screened interaction are negligible. Thus, in this limit the damping rate of hot electrons is obtained from Eq. (11) with use of the RPA dielectric function.

Now we focus on the scattering of hot electrons just above the Fermi level, i. e., $E-E_{\mathrm{F}} \ll E_{\mathrm{F}}$. As the energy transfer $\omega$ cannot exceed the value $E-$ $E_{\mathrm{F}}$, the frequency entering $\operatorname{Im}\left[\epsilon_{q, \omega}^{-1}\right]$ is always small, one can take

$\operatorname{Im}\left[-\epsilon_{q, \omega}^{-1}\right]=\frac{\operatorname{Im}\left[\epsilon_{q, \omega}\right]}{\left|\epsilon_{q, 0}\right|^{2}} \rightarrow \frac{2}{q^{3}} \epsilon_{q, 0}^{-2} \omega$,

and the $\left(E-E_{\mathrm{F}}\right)^{2}$ quadratic scaling of the hot-electron damping rate is predicted. If one further replaces, within the high-density limit $\left(q_{\mathrm{F}} \rightarrow \infty\right)$, the static dielectric function $\epsilon_{q, 0}$ by the Thomas-Fermi approximation, and extends, at the same time, the maximum momentum transfer $\left(q \sim 2 q_{\mathrm{F}}\right)$ to infinity, then one finds

$\tau^{-1}=\frac{\left(\pi / q_{\mathrm{F}}\right)^{3 / 2}}{16} \frac{\left(E-E_{\mathrm{F}}\right)^{2}}{k_{i}}$.

If we replace $k_{i} \rightarrow q_{\mathrm{F}}$ in Eq. (36), then the damping rate of Quinn and Ferrell [38] is obtained, $\tau_{\mathrm{QF}}^{-1}$, as given by Eq. (C.8). For the lifetime, one writes 6

$\tau_{\mathrm{QF}}=263 r_{s}^{-5 / 2}\left(E-E_{\mathrm{F}}\right)^{-2} \mathrm{fseV}^{2}$.

In Eq. (35), Im $\epsilon_{q, \omega}$ represents a measure of the number of states available for real transitions, whereas the denominator $\left|\epsilon_{q, 0}\right|^{2}$ accounts for the screening in the interaction between the hot electron and the Fermi sea. Hence, the hot-electron lifetime is determined by the competition between transitions and screening. Though increasing the electron density makes the density of states (DOS) larger, momentum and energy conservation prevents, in the case of a FEG, the sum over available states from any dependence on $r_{s}$, as shown by Eq. (35). As a result, the scattering rate of hot electrons in a FEG only depends on the electron-density parameter through the screening and the initial momentum $\boldsymbol{k}_{i}$.

\footnotetext{
${ }^{6} 1 \mathrm{au}=658 \mathrm{meV}$ fs.
}

High densities make the interaction weaker [the integration of $\left|\epsilon_{q, \omega}\right|^{-2}$ scales, in the high-density limit, as $\left.q_{\mathrm{F}}^{-3 / 2}\right]$ and momenta of excited electrons larger $\left[1 / k_{i} \rightarrow q_{\mathrm{F}}^{-1}\right]$, which results in the $r_{s}^{-5 / 2}$ scaling described by Eq. (37).

In Fig. 3 we represent the ratio $\tau / \tau_{\mathrm{QF}}$, versus $E-E_{\mathrm{F}}$, for an electron density equal to that of valence electrons in copper $\left(r_{s}=2.67\right)$, as obtained from Eq. (11) with the full RPA dielectric function (solid line) and from Eq. (36) (dashed line). Though in the limit $E \rightarrow E_{\mathrm{F}}$ the available phase space for real transitions is simply $E-E_{\mathrm{F}}$, which yields the $\left(E-E_{\mathrm{F}}\right)^{2}$ quadratic scaling of Eqs. (36) and (37), as the energy increases momentum and energy conservation prevents the available phase space from being as large as $E-E_{\mathrm{F}}$. Hence, the actual lifetime departures from the $k_{i} /\left(E-E_{\mathrm{F}}\right)^{2}$ scaling predicted for electrons in the vicinity of the Fermi surface, differences between full RPA calculations (solid line) and the results predicted by Eq. (36) (dashed line) ranging from $\sim 2 \%$ at $E \approx E_{\mathrm{F}}$ to $\sim 35 \%$ at $E-E_{\mathrm{F}}=5$ $\mathrm{eV}$. For comparison, also represented in this figure is the ratio $\tau / \tau_{\mathrm{QF}}$ obtained from the approximations of Eq. (C.4) (dotted line) and Eq. (C.7) (dashed-dotted line).

The result of going beyond the RPA has been discussed by various authors [40,44-50]. In an early

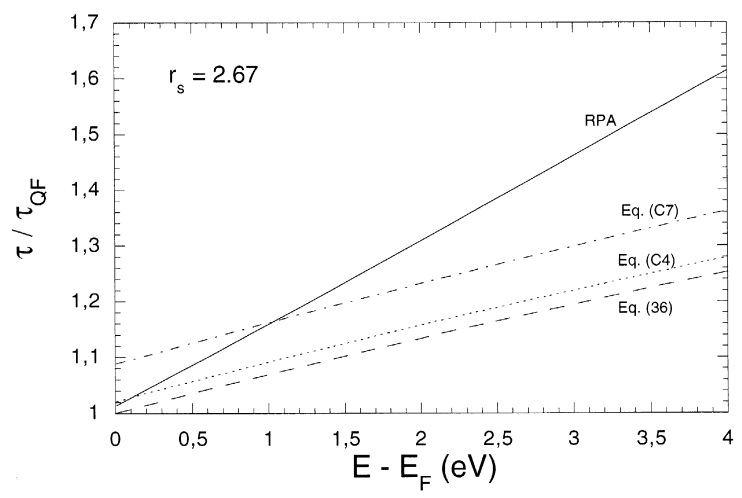

Fig. 3. Ratio $\tau / \tau_{\mathrm{QF}}$ between the lifetime $\tau$ evaluated in various approximations and the lifetime $\tau_{\mathrm{QF}}$ of Eq. (37), versus $E-E_{\mathrm{F}}$, as obtained for hot electrons in a homogeneous electron gas with $r_{s}=2.67$. The solid line represents the result obtained from Eq. (11), within RPA. Results obtained from Eqs. (C.4) and (C.7) are represented by dotted and dashed-dotted lines, respectively. The dashed line represents the result obtained from Eq. (36). 


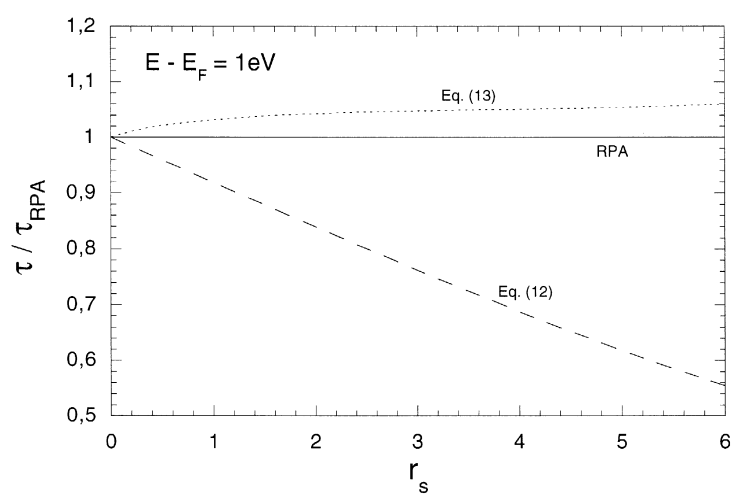

Fig. 4. Exchange and correlation effects on the lifetime of hot electrons with $E-E_{\mathrm{F}}=1 \mathrm{eV}$. The dashed line represents, as a function of $r_{s}$, the ratio between lifetimes derived from Eq. (11) with use of the dielectric function of Eq. (12) with $\left(G_{q, \omega} \neq 0\right)$ and without $\left(G_{q, \omega}=0\right)$ local-field corrections. The dotted line represents, as a function of $r_{s}$, the ratio between lifetimes derived from Eq. (11) with use of the dielectric function of Eq. (13) with $\left(G_{q, \omega} \neq 0\right)$ and without $\left(G_{q, \omega}=0\right)$ local-field corrections. If the local-field factor $G_{q, \omega}$ is taken to be zero, both Eqs. (12) and (13) give the same result (solid line).

paper, Ritchie and Ashley [40] investigated the simplest exchange process in the scattering between the probe electron and the electron gas. Though this exchange contribution to the e-e scattering rate is of a higher order in the electron-density parameter $r_{s}$ than the direct term, it was found to yield, for $r_{s}=2.07$ and $E \sim E_{\mathrm{F}}$, a $\sim 70 \%$ increase with respect to the RPA lifetime, and an even larger increase in the case of metals with $r_{s}>2$. This reduction of the e-e scattering rate appears as a consequence of the exclusion principle keeping two electrons of parallel spin away from the same point, thereby reducing their effective interaction.

Neither the effect of Coulomb correlations between the probe electron and the electron gas, which also influence the e-e mutual interaction, nor $\mathrm{XC}$ effects between pairs of electrons within the Fermi sea were included by Ritchie and Ashley [40]. Kleinman [44] included not only XC between the incoming electron and an electron from the Fermi sea but also $\mathrm{XC}$ between pairs of electrons within the Fermi sea, and found a result which reduced the $\sim 70 \%$ increase obtained by Ritchie and Ashley for Al to a $\sim 1 \%$ increase. Alternative approximations for the $\mathrm{XC}$ corrected $\mathrm{e}-\mathrm{e}$ interaction were derived by Penn [45] and by Kukkonen and Overhauser [46]. From an evaluation of the test-charge-electron dielectric function of Eq. (13) and with use of a static local-field factor, Penn [47] concluded that the introduction of exchange and correlation has little effect on the lifetime of hot electrons, in agreement with early calculations by Kleinman [44].

As we are interested in the low-frequency $(\omega \rightarrow 0)$ behaviour of the electron gas, we can safely approximate the local-field factor by the static limit, $G_{q, 0}$, which we choose to be given by Eq. (A.15). Our results, as obtained from Eq. (11) with the dielectric function of either Eq. (12) or Eq. (13) are presented in Figs. 4 and 5 by dashed and dotted lines, respectively, as a function of $r_{s}$ for hot electrons with $E-E_{\mathrm{F}}=1 \mathrm{eV}$ (Fig. 4), and as a function of $E-E_{\mathrm{F}}$ with $r_{s}=2.67$ (Fig. 5). Solid lines represent RPA calculations, as obtained with the local-field factor $G_{q, \omega}$ set equal to zero. We note from these figures that local-field corrections in the screening reduce the lifetime of hot electrons in a FEG with an electron density equal to that of valence $\left(4 s^{1}\right)$ electrons in $\mathrm{Cu}\left(r_{s}=2.67\right)$ by $\sim 20 \%$. However, this reduction is slightly more than compensated by the large enhancement of the lifetime produced by the existence of local-field corrections in the interaction between the probe electron and the electron gas. As a consequence, RPA calculations (solid line) produce lifetimes that are shorter than more realistic results obtained with full inclusion of XC effects (dotted line) by $\sim 5 \%$.

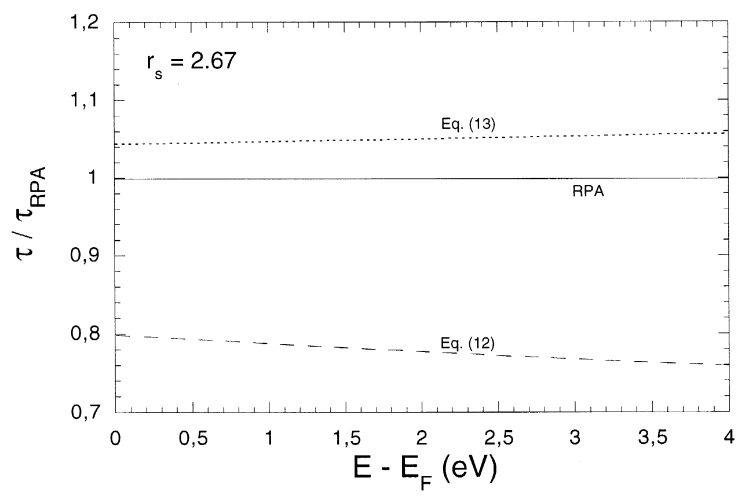

Fig. 5. As in Fig. 4, but for hot electrons in a homogeneous electron gas with $r_{s}=2.67$, and as a function of the electron energy $E-E_{\mathrm{F}}$ with respect to the Fermi level. 
Instead of calculating the damping rate $\tau^{-1}$ onthe-energy-shell ( $E=\omega_{\boldsymbol{k}_{\mathrm{k}}}$ ), Lundqvist [48] expanded the electron self-energy in the deviation of the actual excitation energy $E$ from the independent-particle result, showing that near the energy-shell $\left(E \sim \omega_{k_{i}}\right)$ interactions renormalize the damping rate by the so-called renormalization constant $Z_{\boldsymbol{k}_{i}}$. Based on Lundqvist's calculations, Shelton [49] derived IMFPs for various values of $r_{s}$ and for electrons with energies between $E_{\mathrm{F}}$ and $\sim 25 E_{\mathrm{F}}$. The resulting IMFPs were larger than those obtained by Quinn [41] by roughly 5-20\%, depending on $r_{s}$ and the electron energy.

In the case of excited electrons near the Fermi level the renormalization constant, as obtained within the GW-RPA, is nearly real and $k$-independent. In the metallic density range $\left(r_{s} \sim 2-6\right)$ one finds $Z \sim$ $0.8-0.7$, and the resulting lifetimes are, therefore, larger than those obtained from Eq. (11) by $\sim 20 \%$.

\subsection{Statistical approximations}

In order to account for the inelastic scattering rates of non-free-electron materials, Tung et al. [54] applied a statistical approximation first developed by Lindhard et al. [106]. This approximation is based on the assumption that the inelastic electron scattering of electrons in a small volume element $\mathrm{d} \boldsymbol{r}$ at $\boldsymbol{r}$ is the same as that of electrons in a FEG with density equal to the local density.

Within the statistical approximation of Ref. [54], for a given density distribution $n(\boldsymbol{r})$ one finds the total scattering rate $\tau^{-1}$ by averaging the corresponding local quantity $\tau^{-1}[n(\boldsymbol{r})]$ over the volume $\Omega$ of the solid:

$$
\left\langle\tau^{-1}\right\rangle=\Omega^{-1} \int \mathrm{d} \boldsymbol{r} \tau^{-1}[n(\boldsymbol{r})] .
$$

By calculating spherically symmetric electron density distributions $n(r)$ in a Wigner-Seitz cell [107], the total scattering rate is obtained from

$$
\left\langle\tau^{-1}\right\rangle=4 \pi \Omega_{W S}^{-1} \int_{0}^{r_{W S}} \mathrm{~d} r r^{2} \tau^{-1}[n(r)],
$$

where $\Omega_{W S}$ and $r_{W S}$ represent the volume and the radius of the Wigner-Seitz sphere of the solid.

Alternatively, following the idea of using optical data in IMFP calculations [5], a number of ap- proaches were developed [55-58] to compute a model energy-loss function $\operatorname{Im}\left[-\epsilon_{q, \omega}^{-1}\right]$ for real solids and then obtain inelastic scattering rates from Eq. (11). In these approaches the model energy-loss function is set in the limit of zero wave vector equal to the imaginary part of the measured optical inverse dielectric function [108], $\operatorname{Im}\left[-1 / \epsilon_{\omega}^{\mathrm{opt}}\right]$, and it is then extended into the non-zero wave vector region by a physically motivated recipe.

Combining the statistical method of Ref. [54] with the use of optical data, Penn [59] developed an improved algorithm to evaluate the dielectric function of the material. The Penn algorithm is based on a model dielectric function in which the momentum dependence is determined by averaging the energyloss function of a FEG, $\operatorname{Im}\left[-1 / \epsilon_{q, \omega}^{\mathrm{FEG}}\right]$, as follows

$\operatorname{Im}\left[-\epsilon_{q, \omega}^{-1}\right]=\int_{0}^{\infty} \mathrm{d} \omega_{p} G\left(\omega_{p}\right) \operatorname{Im}\left[1 / \epsilon_{q, \omega}^{\mathrm{FEG}}\left(\omega_{p}\right)\right]$,

where

$G(\omega)=\frac{2}{\pi \omega} \operatorname{Im}\left[-1 / \epsilon_{\omega}^{\mathrm{op}}\right]$.

The Penn algorithm has been employed by Tanuma et al. [60] to calculate IMFPs for 50 to 2000 $\mathrm{eV}$ in a variety of materials comprising elements, inorganic compounds, and organic compounds. Recently, several other groups [61] have calculated IMFPs from optical data in a manner similar to that proposed by Penn [59], with some differences in approach, and high-energy IMFPs now seem to be well understood [62].

The effect of $d$-electrons in noble metals has been recently investigated by Zarate et al. [109], by including the $d$-band contribution to the measured optical inverse dielectric function into a FEG description of the $s-p$ part of the response.

In order to account for the actual DOS in real materials, early IMFP calculations were carried out by Krolikowski and Spicer [53], with the explicit assumption that the matrix elements of the screened e-e interaction entering Eq. (5) are momentum independent. This so-called "random" $k$ approximation $[110,111]$ has proved to be useful in cases where the DOS plays a key role in the determination of scattering rates, as in the case of ferromagnetic materials 
$[112,113]$, thereby allowing to explain the existence of spin-dependent hot-electron lifetimes [26,27]. Although this method, due to its simplicity, cannot provide full quantitative agreement with the experiment, it provides a useful tool for the analysis of experimental data, thus allowing to isolate the effects that are directly related to the DOS.

Figs. 6 and 7 show the lifetime versus energy, for representative free-electron-like and non-free-electron-like materials, Al (Fig. 6) and $\mathrm{Cu}$ (Fig. 7), respectively. First of all, we consider a relatively free-electron-like solid such as $\mathrm{Al}$ (see Fig. 6). The contribution to the inelastic scattering of low-energy electrons in $\mathrm{Al}$ coming from the excitation of core electrons is negligible. Hence, statistical approximations yield results that nearly coincide with the FEG calculation with $r_{s}=2.07$. However, the effective number of valence electrons in $\mathrm{Al}$ is 3.1 rather than 3 (the actual number of valence electrons), and lifetimes calculated from the statistical model of Ref. [54] are, therefore, slightly larger than those obtained within a FEG description. At higher energies, new contributions to the inelastic scattering come from the excitation of core electrons, and FEG lifetimes would, therefore, be much longer than those obtained from the more realistic statistical approximations.

For non-free-electron-like materials such as $\mathrm{Cu}$, the role of $d$ states in the electron relaxation process

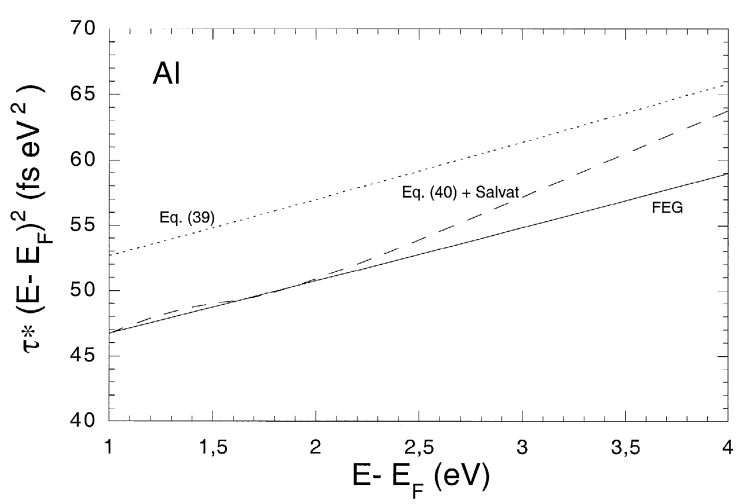

Fig. 6. Averaged lifetimes of hot electrons in $\mathrm{Al}$, versus $E-E_{\mathrm{F}}$, as obtained from Eq. (39) with the local electron density of Ref. [54] (dotted line), and from Eq. (11) with the model dielectric function of Eq. (40) and the recipe described by Salvat et al. [58] to obtain the optical energy-loss function (dashed line). The solid line represents the result obtained from Eq. (11) with use of the free-electron gas RPA energy-loss function and $r_{s}=2.07$.

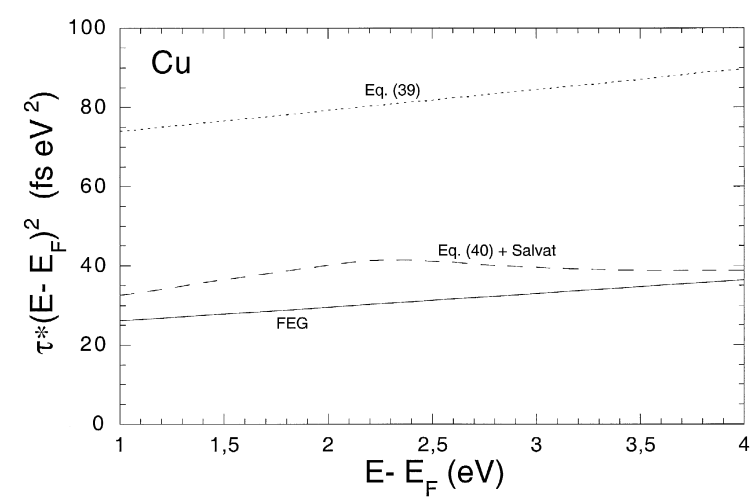

Fig. 7. Averaged lifetimes of hot electrons in $\mathrm{Cu}$, versus $E-E_{\mathrm{F}}$, as obtained from Eq. (39) with the local electron density of Ref. [54] (dotted line), and from Eq. (11) with the model dielectric function of Eq. (40) and the recipe described by Salvat et al. [58] to obtain the optical energy-loss function (dashed line). The solid line represents the result obtained from Eq. (11) with use of the free-electron gas RPA energy-loss function and $r_{s}=2.67$.

is of crucial importance, even in the case of verylow-energy electrons. The effective number of valence electrons in $\mathrm{Cu}$ that contribute through the average of Eq. (39), at low electron energies, to the inelastic scattering ranges from $\sim 2.5$ far from atomic positions to $\sim 7.5$ in a region where the binding energy is already too large. Since an enhanced electron density results in a stronger screening and, therefore, a longer lifetime (see, e.g., Eq. (37)), the statistical approximation yields lifetimes that are longer than those obtained within a FEG model with the electron density equal to that of valence $\left(4 s^{1}\right)$ electrons in $\mathrm{Cu}\left(r_{s}=2.67\right)$, but shorter than those obtained within a FEG model with the electron density equal to that of all $4 s^{1}$ and $3 d^{10}$ electrons in $\mathrm{Cu}$. We note that the theory of Penn [59] gives shorter lifetimes than the theory of Tung et al. [54], which is the result of spurious contributions to the average energy-loss function of Eq. (40) from $\operatorname{Im}\left[1 / \epsilon_{\omega}^{\mathrm{opt}}\right]$ at very low-frequencies.

For comparison with the 'universal' relationship $\tau^{-1}=0.13\left(E-E_{\mathrm{F}}\right)$ proposed by Goldmann et al. [10] for $\mathrm{Cu}$, on the basis of experimental angle-resolved inverse photoemission spectra, lifetime-widths $\tau^{-1}$ of high-energy electrons in $\mathrm{Cu}$ are represented in Fig. 8. Solid and dashed-dotted lines represent results obtained from Eqs. (11) and (39). Dashed and dashed-dotted-dotted-dotted lines represent the result 


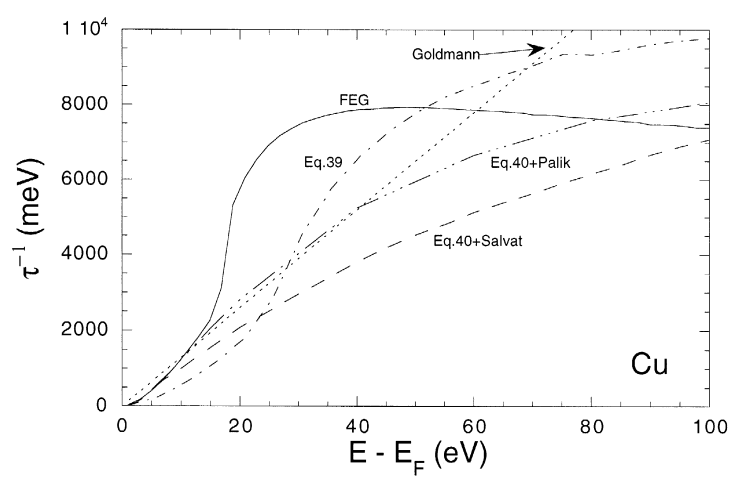

Fig. 8. Averaged lifetime-widths $\tau^{-1}$ of excited electrons in $\mathrm{Cu}$, versus $E-E_{\mathrm{F}}$, as obtained from Eq. (39) with the local electron density of Ref. [54] (dotted line), and from Eq. (11) with the model dielectric function of Eq. (40) and the recipe described by Salvat et al. [58] to obtain the optical energy-loss function (dashed line). The dashed-dotted-dotted-dotted line represents the result obtained from Eq. (11) with use of the model dielectric function of Eq. (40) and the experimental optical energy-loss function of Ref. [108]. The dotted line represents the result of using the 'universal' relationship $\tau^{-1}=0.13\left(E-E_{\mathrm{F}}\right)$ proposed by Goldmann et al. [10].

of introducing into Eq. (11) the model energy-loss function of Eq. (40) with either the recipe described by Salvat et al. (dashed line) or with the measured optical response function taken from Ref. [108] (dashed-dotted-dotted-dotted line), and the empirical formula of Goldmann et al. [10] is represented by a dotted line. We note from this figure that while at low electron energies $\tau^{-1}$ increases quadratically with $E-E_{\mathrm{F}}$, a combination of inner-shell and plasmon contributions results in lifetime-widths that approximately reproduce, for electron energies in the range $\sim 10-50 \mathrm{eV}$ above the Fermi level, the empirical prediction [10] that the lifetime-width increases linearly with increasing distance from $E_{\mathrm{F}}$.

High-energy lifetime-widths and IMFPs seem to be well described by model dielectric functions, by assuming that the probe wave functions are simply plane waves. Nevertheless, in the case of low-energy electrons band structure effects are found to be important, even in the case of free-electron-like metals such as $\mathrm{Al}$, and a a treatment of the electron dynamics that fully includes band structure effects is necessary for quantitative comparisons with the experiment.

\subsection{First-principles calculations}

$\mathrm{Ab}$ initio calculations of the inelastic lifetime of hot electrons in metals have been carried out only very recently $[63,64]$. In this work $[63,64]$, Bloch states were first expanded in a plane-wave basis, and the Kohn-Sham equation of density-functional theory (DFT) $[114,115]$ was then solved by invoking the local-density approximation (LDA). The electron-ion interaction was described by means of a non-local, norm-conserving ionic pseudopotential [116], and the one-electron Bloch states were then used to evaluate both the $B_{i f}$ coefficients and the dielectric matrix $\epsilon_{G, G^{\prime}}$ entering Eq. (27).

First-principles calculations of the average lifetime $\tau(E)$ of hot electrons in real $\mathrm{Al}$, as obtained from Eq. (27) with full inclusion of crystalline local field effects, are presented in Fig. 9 by solid circles. As Al crystal does not present strong electron-density gradients nor special electron-density directions (bondings), contributions from the so-called crystalline local-field effects are found to be negligible. On the other hand, band-structure effects on the imaginary part of the inverse dielectric matrix are approximately well described with the use of a statistical approximation, as obtained from Eq. (39) (dotted

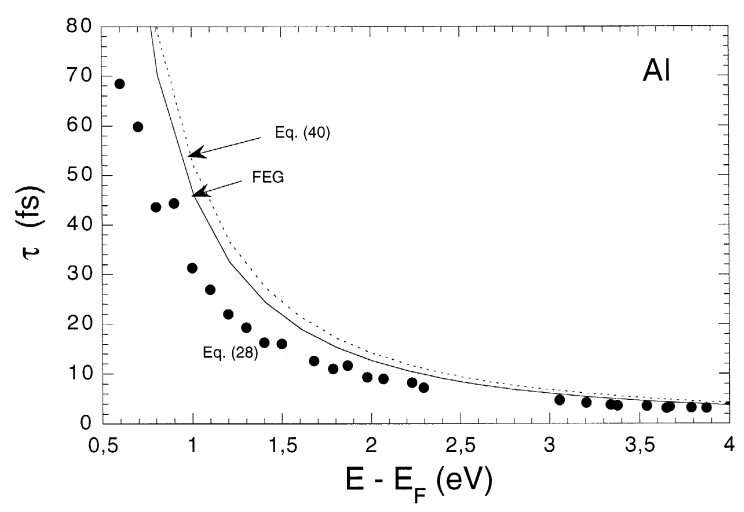

Fig. 9. Hot-electron lifetimes in Al. Solid circles represent the full ab initio calculation of $\tau(E)$, as obtained after averaging $\tau$ of Eq. (27) over wave vectors and over the band structure for each wave vector. The solid line represents the lifetime of hot electrons in a FEG with $r_{s}=2.07$, as obtained from Eq. (11). The dotted line represents the statistically averaged lifetime, as obtained from Eq. (39) by following the procedure of Tung et al. [54]. 
line), thereby resulting in lifetimes that are just slightly larger than those of hot electrons in a FEG with $r_{s}=2.07$ (solid line). Therefore, differences between full ab initio calculations (solid circles) and FEG calculations (solid line) are mainly due to the sensitivity of hot-electron initial and final wave functions on the band structure of the crystal. When the hot-electron energy is well above the Fermi level, these orbitals are very nearly plane-wave states and the lifetime is well described by FEG calculations. However, in the case of hot-electron energies near the Fermi level, initial and final states strongly depend on the actual band structure of the crystal. Due to the opening, at these energies, of interband transitions, band structure effects tend to decrease the inelastic lifetime by a factor that varies from $\sim 0.65$ near the Fermi level $\left(E-E_{\mathrm{F}}=1 \mathrm{eV}\right)$ to a factor of $\sim 0.75$ for $E-E_{\mathrm{F}}=3 \mathrm{eV}$.

Ab initio calculations of the average lifetime $\tau(E)$ of hot electrons in real $\mathrm{Cu}$, the most widely studied metal by TR-2PPE, are exhibited in Fig. 10 by solid

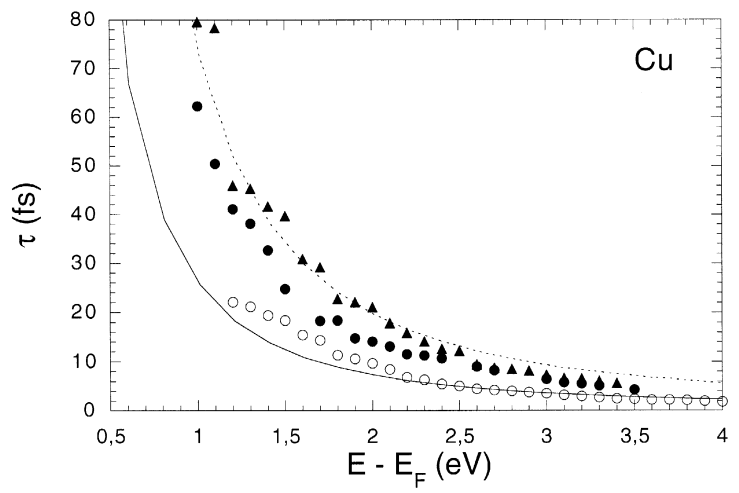

Fig. 10. Hot-electron lifetimes in $\mathrm{Cu}$. Solid circles represent the full ab initio calculation of $\tau(E)$, as obtained after averaging $\tau$ of Eq. (27) over wave vectors and over the band structure for each wave vector. The solid line represents the lifetime of hot electrons in a FEG with $r_{s}=2.67$, as obtained from Eq. (11). The dotted line represents the statistically averaged lifetime, as obtained from Eq. (39) by following the procedure of Tung et al. [54]. Open circles represent the result obtained from Eq. (33) by replacing hot-electron initial and final states in $\left|B_{i f}(\boldsymbol{q}+\boldsymbol{G})\right|^{2}$ by plane waves and the dielectric function in $\left|\epsilon_{G, G}(\boldsymbol{q}, \omega)\right|^{-2}$ by that of a FEG with $r_{s}=2.67$, but with full inclusion of the band structure in the calculation of $\operatorname{Im}\left[\epsilon_{G, G}(q, \omega)\right]$. Full triangles represent the result obtained from Eq. (33) by replacing hot-electron initial and final states in $\left|B_{i f}(\boldsymbol{q}+\boldsymbol{G})\right|^{2}$ by plane waves, but with full inclusion of the band structure in the evaluation of both $\operatorname{Im}\left[\epsilon_{G, G}(q, \omega)\right]$ and $\left|\epsilon_{G, G}(q, \omega)\right|^{-2}$. circles, as obtained from Eq. (27) with full inclusion of crystalline local field effects and by keeping all $4 s^{1}$ and $3 d^{10}$ Bloch states as valence electrons in the pseudopotential generation. The lifetime of hot electrons in a FEG with the electron density equal to that of valence $\left(4 s^{1}\right)$ electrons in $\mathrm{Cu}\left(r_{s}=2.67\right)$ is represented by a solid line, and the statistically averaged lifetime, as obtained from Eq. (39), is represented by a dotted line. These calculations indicate that the lifetime of hot electrons in real $\mathrm{Cu}$ is, within RPA, larger than that of electrons in a FEG with $r_{s}=2.67$, this enhancement varying from a factor of $\sim 2.5$ near the Fermi level $\left(E-E_{\mathrm{F}}=1.0 \mathrm{eV}\right)$ to a factor of $\sim 1.5$ for $E-E_{\mathrm{F}}=3.5 \mathrm{eV}$. Ab initio calculations of the lifetime of hot electrons in $\mathrm{Cu}$, obtained by just keeping the $4 s^{1}$ Bloch states as valence electrons in the pseudopotential generation, were also performed, and they were found to nearly coincide with the FEG calculations. Hence, $d$-band states play a key role in the hot-electron decay mechanism.

In order to address the various aspects of the role that localized $d$-bands play on the lifetime of hot electrons in $\mathrm{Cu}$, now we neglect crystalline local-field effects and present the result of evaluating hot-electron lifetimes from Eq. (33). First, we replace hotelectron initial and final states in $\left|B_{i f}(\boldsymbol{q}, \boldsymbol{G})\right|^{2}$ by plane waves, and the dielectric function in $\left|\epsilon_{G, G}(q, \omega)\right|^{-2}$ by that of a FEG with $r_{s}=2.67$. If we further replaced $\operatorname{Im}\left[\epsilon_{G, G}(q, \omega)\right]$ by that of a FEG, i.e., Eq. (8), then we would obtain the FEG calculation represented by a solid line. Instead, Ogawa et al. [18] included the effect of $d$-bands on the lifetime by computing the actual number of states available for real transitions, within Eq. (8), from the band structure of $\mathrm{Cu}$, and they obtained a result that is for $E-E_{\mathrm{F}}>2 \mathrm{eV}$ well below the FEG calculation ${ }^{7}$. However, if one takes into account, within

\footnotetext{
${ }^{7}$ These authors [18] approximated the FEG dielectric function in $\left|\epsilon_{G, G}(q, \omega)\right|^{-2}$ within the static Thomas-Fermi model, as in Eq. (C.6), and with the screening length $q_{\mathrm{TF}}^{-1}=0.47 \AA$ taken from the actual DOS at the Fermi level. Though the actual DOS at the Fermi level being larger than the corresponding DOS from the FEG model makes the screening stronger $\left(q_{\mathrm{TF}}>q_{\mathrm{TF}}^{\mathrm{FEG}}, q_{\mathrm{TF}}^{\mathrm{FEC}}\right.$ being the screening length from the FEG model), the increase in the actual number of states available for real transitions yields lifetimes that are below the FEG calculation.
} 
a full description of the band structure of the crystal in the evaluation of $\operatorname{Im}\left[\epsilon_{G, G}(\boldsymbol{q}, \omega)\right]$ (see Eqs. (29) and (31)), couplings between the states participating in real transitions, then one obtains the result represented in Fig. 10 by open circles. Since the states just below the Fermi level, which are available for real transitions, are not those of free-electron states, localization results in lifetimes of hot electrons with $E-E_{\mathrm{F}}<2 \mathrm{eV}$ (open circles) that are slightly larger than predicted within the FEG model of the metal. At larger energies this band-structure calculation (open circles) predicts a lower lifetime than within the FEG model, due to opening of the $d$-band scattering channel dominating the DOS with energies from $\sim 2 \mathrm{eV}$ below the Fermi level. Thus, this calculation shows at $E-E_{\mathrm{F}} \sim 2 \mathrm{eV}$ a slight deviation from the quadratic scaling predicted within the FEG model, in qualitative agreement with experimentally determined decay times in $\mathrm{Cu}$.

While the excitation of $d$ electrons diminishes the lifetime of hot electrons with energies $E-E_{\mathrm{F}}>2$ $\mathrm{eV}, d$ electrons also give rise to additional screening, thus increasing the lifetime of all hot electrons above the Fermi level. That this is the case is obvious from the band-structure calculation exhibited by full triangles in Fig. 10. This calculation is the result obtained from Eq. (33) by still replacing hot-electron initial and final states in $\left|B_{0 f}(\boldsymbol{q}+\boldsymbol{G})\right|^{2}$ by plane waves (plane-wave calculation) but including the full band structure of the crystal in the evaluation of both $\operatorname{Im}\left[\boldsymbol{\epsilon}_{\boldsymbol{G}, \boldsymbol{G}}(\boldsymbol{q}, \omega)\right]$ and $\left|\boldsymbol{\epsilon}_{\boldsymbol{G}, \boldsymbol{G}}(\boldsymbol{q}, \omega)\right|^{-2}$. The effect of virtual interband transitions giving rise to additional screening is to increase, for hot-electron energies under study, the lifetime by a factor of $\approx 3$, in qualitative agreement with the approximate prediction of Quinn [43] and with the use of the statistical average of Ref. [54].

Finally, band-structure effects on hot-electron energies and wave functions are investigated. Full band-structure calculations of Eq. (27) with and without (see also Eq. (33)) the inclusion of crystalline local field corrections were carried out [64], and these corrections were found to be negligible for $E-E_{\mathrm{F}}>1.5 \mathrm{eV}$, while for energies very near the Fermi level neglection of these corrections resulted in an overestimation of the lifetime of less than $5 \%$. Therefore, differences between the full (solid circles) and plane-wave (solid triangles) band-structure cal- culations come from the sensitivity of hot-electron initial and final states on the band structure of the crystal. When the hot-electron energy is well above the Fermi level, these states are very nearly planewave states for most of the orientations of the wave vector, and the lifetime is well described by planewave calculations (solid circles and triangles nearly coincide for $E-E_{\mathrm{F}}>2.5 \mathrm{eV}$ ). However, in the case of hot-electron energies near the Fermi level initial and final states strongly depend on the orientation of the wave vector and on the shape of the Fermi surface. For most orientations, flattening of the Fermi surface tends to increase the hot-electron decay rate [42], while the existence of the so-called necks on the Fermi surface of noble metals results in very small scattering rates for a few orientations of the wave vector. After averaging $\tau^{-1}(\boldsymbol{k}, n)$ over all orientations, Fermi surface shape effects tend to decrease the inelastic lifetime.

Scaled lifetimes, $\tau \times\left(E-E_{\mathrm{F}}\right)^{2}$, of hot electrons in $\mathrm{Cu}$ are represented in Fig. 11, as a function of $E-E_{\mathrm{F}}$. Results obtained, within RPA, from Eqs.

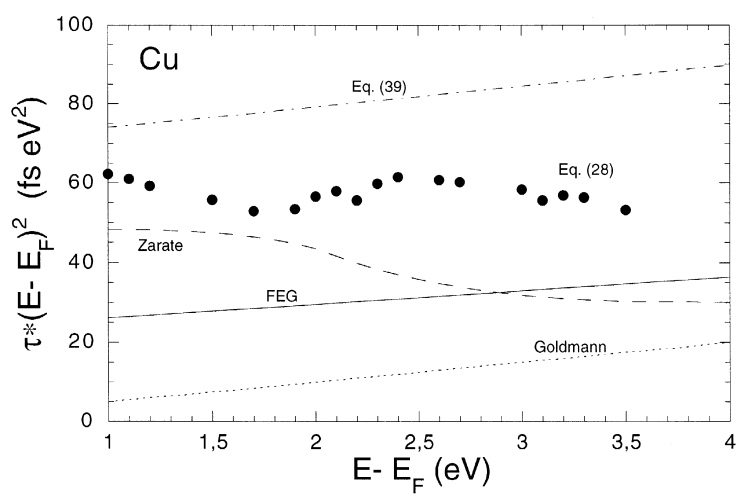

Fig. 11. Scaled hot-electron lifetimes in $\mathrm{Cu}$. Solid circles represent the full ab initio calculation of $\tau(E)$, as obtained after averaging $\tau$ of Eq. (27) over wave vectors and over the band structure for each wave vector. The solid line represents the lifetime of hot electrons in a FEG with $r_{s}=2.67$, as obtained from Eq. (11). The dasheddotted line represents the statistically averaged lifetime, as obtained from Eq. (39) by following the procedure of Tung et al. [54]. The dashed line represents the result of following the procedure described in Ref. [109], and the dotted line is the result of using the 'universal' relationship $\tau^{-1}=0.13\left(E-E_{\mathrm{F}}\right)$ proposed by Goldmann et al. [10]. 
(11) and (39) are represented by solid and dasheddotted lines, respectively, the ab initio calculations of Ref. [64] are represented by solid circles, and the dashed line represents the calculations described in Ref. [109]. These model calculations [109] show that above the $d$-band threshold, at $\sim-2 \mathrm{eV}$ relative to the Fermi level, $d$-band electrons can only participate in the screening, thereby producing longer lifetimes, while at larger energies lower lifetimes are expected, due to opening of the $d$-band scattering channel that dominates the DOS with energies $\sim 2$ $\mathrm{eV}$ below the Fermi level. For comparison, the empirical formula proposed by Goldmann et al. is represented by a dotted line.

Scaled lifetimes of hot electrons in $\mathrm{Cu}$, determined from a variety of experiments [17-20], are represented in Fig. 12, as a function of $E-E_{\mathrm{F}}$. Though there are large discrepancies among results obtained in different laboratories, most experiments give lifetimes that are considerably longer than predicted within a free-electron description of the metal, in agreement with first-principles calculations. Measurements of hot-electron lifetimes have also been performed for other noble and transition metals [16,17,22-24], simple metals [25], ferromagnetic

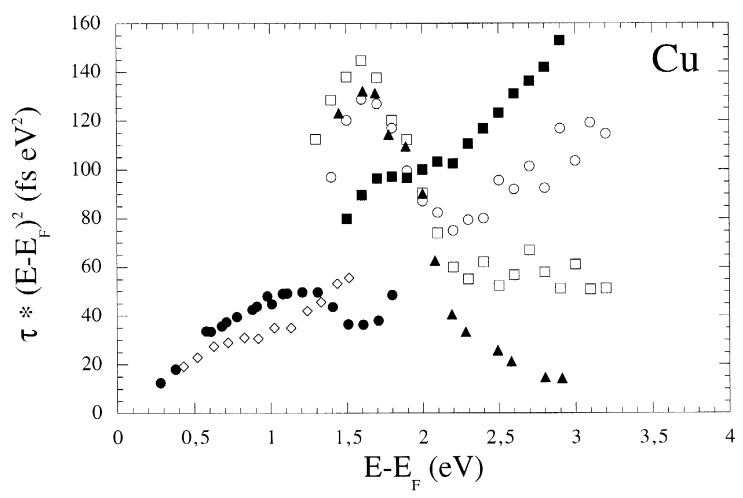

Fig. 12. Experimental lifetimes of low-energy electrons in $\mathrm{Cu}$, as taken from Knoesel et al. [20] (solid circles), from Ogawa et al. [18] $(\mathrm{Cu}[100]$ : open circles, $\mathrm{Cu}[110]$ : open squares, $\mathrm{Cu}[111]$ : solid squares), from Aeschlimann et al. [17] (solid triangles), and from Cao et al. [19] with $\omega_{\text {photon }}=1.63 \mathrm{eV}$ (open diamonds).
Table 1

Available experimental data for hot-electron lifetimes in metals, as obtained by time-resolved two-photon photoemission and ballistic electron emission microscopy (BEEM)

\begin{tabular}{ll}
\hline Metal & Reference (technique) \\
\hline $\mathrm{Cu}$ & {$[17-20]($ TR-TPPE) } \\
$\mathrm{Ag}$ & {$[16,17]($ TR-TPPE) } \\
$\mathrm{Au}$ & {$[17,22]($ TR-TPPE); $[23]$ (BEEM) } \\
$\mathrm{Ta}$ & {$[16,17](\mathrm{TR}-\mathrm{TPPE})$} \\
$\mathrm{Pd}$ & {$[24](\mathrm{BEEM})$} \\
$\mathrm{Al}$ & {$[25](\mathrm{TR}-\mathrm{TPPE})$} \\
$\mathrm{Co}$ & {$[26,27](\mathrm{TR}-\mathrm{TPPE})$} \\
$\mathrm{Fe}$ & {$[27](\mathrm{TR}-\mathrm{TPPE})$} \\
\hline
\end{tabular}

solids [26,27], and high- $T_{\mathrm{c}}$ superconductors [28] (see Table 1).

\section{Lifetimes of image-potential states at metal surfaces}

\subsection{Concept and development of image states}

A metal surface generates electron states that do not exist in a bulk metal. These states can be classified into two groups, according to their charge density localization relative to the surface atomic layer: intrinsic surface states and image-potential states. The so-called intrinsic surface states, predicted by Tamm [117] and Shockley [118], are localized mainly at the surface atomic layer. Image-potential states [68-75] appear in the vacuum region of metal surfaces with a band gap near the vacuum level, as a result of the self-interaction of the electron with the polarization charge it induces at the surface. Far from the surface, into the vacuum, this potential well approaches the long-range classical image potential, $-1 / 4 z, z$ being the distance from the surface, and it gives rise to a series of image-potential states localized outside the metal.

In a hydrogenic model, with an infinitely high repulsive surface barrier, these states form a Rydberg-like series with energies [69]

$E_{n}=\frac{-0.85 \mathrm{eV}}{n^{2}}$

converging towards the vacuum level $E_{v}=0$. The corresponding eigenfunctions are given by the radial solutions of an $s$-like state of the hydrogen atom

$\phi_{n}(z) \propto z R_{n}^{l=0}(z / 4)$. 
The lifetime of these states scales asymptotically with the quantum number $n$, as follows [69]

$\tau_{n} \propto n^{3}$.

For a finite repulsive surface barrier, as is the case for real metal surfaces, Eq. (42) may be transformed into

$E_{n}=\frac{-0.85 \mathrm{eV}}{(n+a)^{2}}$,

where $a$ is a quantum defect depending on both the energy-gap position and width and also on the position of the image state relative to the gap $[68,69]$.

After demonstration of the resolubility of the image-state series on metal surfaces [69], these states were found experimentally [119-121]. Binding energies of these states have been measured by inverse photoemission (IPE) [119-122], two-photon photoemission (2PPE) [123-126], and time-resolved twophoton photoemission (TR-2PPE) [29-35]. These measurements have provided highly accurate data of image-state binding energies at the surfaces of many noble and transition metals, as shown, e.g., in Ref. [74]. Along with the measurements of image-state energies, the dispersion of these states has also been measured, and it has been found that only on a few surfaces such as $\operatorname{Ag}(100), \operatorname{Ag}(111)$, and $\mathrm{Ni}(111)$ the first image state is characterized by an effective mass that exceeds the free-electron mass [74]. At the same time, theoretical efforts have been directed to create relatively simple models that reproduce the experimentally observed binding energies and effective masses of image states, and also to evaluate the image-plane position [70-73,127-135]. First-principles calculations of image states have also been carried out [81,136-142], with various degrees of sophistication.

This intensive work on image states has resulted in an understanding of some of the key points of the physics of these states and of the relatively extensive data-base of their energies on noble and transition metals.

\subsection{Lifetimes of image states}

\subsubsection{Introduction}

In contrast to the relatively simple spectroscopic problem of determining the position of spectral fea- tures that directly reflect the density of states and which may be, in principle, calculated within a oneelectron theory, the study of spectral widths or lineshapes is essentially a many-body problem [143]. These spectral widths appear as a result of electronelectron, electron-defect, electron-phonon, and electron-photon interactions [143-147], and they are also influenced by phonon-phonon interactions [31,148]. Accurate and systematic measurements of the linewidth of image states on metal surfaces were carried out with the use of 2PPE spectroscopy (for a review see, e.g., Ref. [74]). These experiments gave smaller values for the image-state lifetime than the ones obtained in recent very-high resolution TR-2PPE measurements [29-35]. The reason for this discrepancy is that the 2PPE linewidth contains not only an energy relaxation contribution (intrinsic lifetime), but also contributions that arise from phase-relaxation processes [147].

The first estimation [69] of the lifetime of image states used simple wave-function arguments to show that the lifetime of image states asymptotically increases with the quantum number $n$, as in Eq. (44). Nearly twenty years later, this prediction was confirmed experimentally for the (100) surface of $\mathrm{Cu}$, for which lifetimes of the first six image states were measured with the use of quantum-beat spectroscopy [33]. The first quantitative evaluation of the lifetime of image states, as obtained within the self-energy formalism, was reported in Ref. [65]. In this calculation hydrogenic-like states were used, with no penetration into the solid, to describe the image-state wave functions, a step model potential was introduced to calculate the bulk final-state wave functions, and a simplified free-electron-gas (FEG) model was utilized to approximate the screened Coulomb interaction. More realistic wave functions, allowing for penetration of the electron into the crystal, were introduced in subsequent calculations [66,67]. In these evaluations the linewidth of the first image state at the $\bar{\Gamma}$ point was shown to be directly proportional to the penetration, and the prediction of Eq. (44) was confirmed.

The penetration of an image state into the bulk is defined as

$p_{n}=\int_{\text {bulk }} \mathrm{d} z \phi_{n}^{*}(z) \phi_{n}(z)$, 
thereby giving a measure of the coupling of this state to bulk electronic states. This coupling, weighted by the screened interaction, is responsible for the decay of image states through electron-hole pair creation. Intuitively, it seems clear that the larger the penetration the stronger the coupling and, therefore, the smaller the lifetime. This idea was exploited to qualitatively explain the linewidth of image states on various surfaces [74], and also the temperature-dependence of the linewidth of the $n=1$ state on $\mathrm{Cu}(111)$ [31]. In this heuristic approximation, the linewidth of an image state is determined by

$$
\Gamma\left(E_{n}\right)=p_{n} \Gamma_{b}\left(E_{n}\right),
$$

where $\Gamma_{b}\left(E_{n}\right)$ is the linewidth of a bulk state corresponding to the energy $E_{n}$. The $\Gamma_{b}\left(E_{n}\right)$ value can be obtained either from first-principles calculations or from the experiment. In many angle-resolved photoemission experiments a linear dependence of the linewidth of bulk $s-p$ and $d$ states is observed for energies in the range 5-50 eV above the Fermi level [7-12],

$$
\Gamma_{b}\left(E_{n}\right)=b\left(E_{n}-E_{\mathrm{F}}\right),
$$

while the linewidth of bulk states in a FEG shows a $\left(E_{n}-E_{\mathrm{F}}\right)^{2}$ quadratic scaling for energies near the Fermi level, as discussed in Section 4.1 and in Appendix C. Image states on noble and transition metal surfaces have energies in the range $4-5 \mathrm{eV}$ above the Fermi level, so that Eqs. (47) and (48) have been applied in Ref. [74], for an estimate of the lifetime broadening, with use of the experimentally determined coefficient $b=0.13$ for $\mathrm{Cu}$ and $\mathrm{Ag}$ [10]. For $\mathrm{Au}$ one also uses $b=0.13$, and for $\mathrm{Ni}$ and $\mathrm{Fe} b$ is taken to be 0.18 [8] and 0.6 [11], respectively.

Recent TR-2PPE measurements have shown that the intrinsic linewidths of the $n=1$ image state on $\mathrm{Cu}(111)$ [31] and $\mathrm{Cu}(100)$ [33,35] are $30 \mathrm{meV}$ and $16.5 \mathrm{meV}$, respectively, while accurate model potential calculations [81] yield penetrations $p_{1}=0.22$ and $p_{1}=0.05$, respectively. Accordingly, imagestate linewidths cannot be explained by simply applying Eq. (47); instead, contributions to the imagestate decaying mechanism coming from either the evanescent tails of bulk states outside the crystal or the existence of intrinsic surface states must also be taken into account, together with an accurate description of surface screening effects. Here we give the results obtained within a theory that incorporates these effects and that has been used recently to evaluate intrinsic linewidths or, equivalently, lifetimes of image states on metal surfaces [77-80].

\subsubsection{Model potential}

It is well known $[69,73,136]$ that image-state wave functions lie mainly in the vacuum side of the metal surface, the electron moving, therefore, in a region with little potential variation parallel to the surface. Hence, these wave functions can be described, with a reasonable accuracy, by using a one-dimensional potential that reproduces the key properties of image states, namely, the position and width of the energy gap and, also, the binding energies of both intrinsic and image-potential surface states at the $\bar{\Gamma}$ point. Such a one-dimensional potential has recently been proposed for a periodic-film model with large vacuum intervals between the solid films $[81,135]$ :

$$
V(z)= \begin{cases}A_{10}+A_{1} \cos \left(2 \pi z / a_{s}\right), & z<D, \\ A_{20}+A_{2} \cos [\beta(z-D)], & D<z<z_{1}, \\ A_{3} \exp \left[-\alpha\left(z-z_{1}\right)\right], & z_{1}<z<z_{i m}, \\ \frac{\exp \left[-\lambda\left(z-z_{i m}\right)\right]-1}{4\left(z-z_{i m}\right)}, & z_{i m}<z,\end{cases}
$$

where the $z$-axis is taken to be perpendicular to the surface. $D$ is the halfwidth of the film, $a_{s}$ is the interlayer spacing, $z_{i m}$ represents the image-plane position, and the origin is chosen in the middle of the film. This one-dimensional potential has ten parameters, $A_{10}, A_{1}, A_{20}, A_{2}, A_{3}, \alpha, \beta, z_{1}, \lambda$, and $z_{i m}$, but only four of them are independent. $A_{10}, A_{1}, A_{2}$, and $\beta$ are chosen as adjustable parameters, the other six parameters being determined from the requirement of continuity of the potential and its first derivative everywhere in space. The parameters $A_{1}$ and $A_{10}$ reproduce the width and position of the energy gap, while $A_{2}$ and $\beta$ reproduce experimental or first-principles energies $E_{0}$ and $E_{1}$ of the $n=0$ $s-p$ like surface state at the $\bar{\Gamma}$ point and the $n=1$ image state, respectively. This potential is shown schematically in Fig. 13. To illustrate the good quality of the image-state wave functions obtained with this model potential, we compare such wave functions with those obtained with the use of first-princi- 


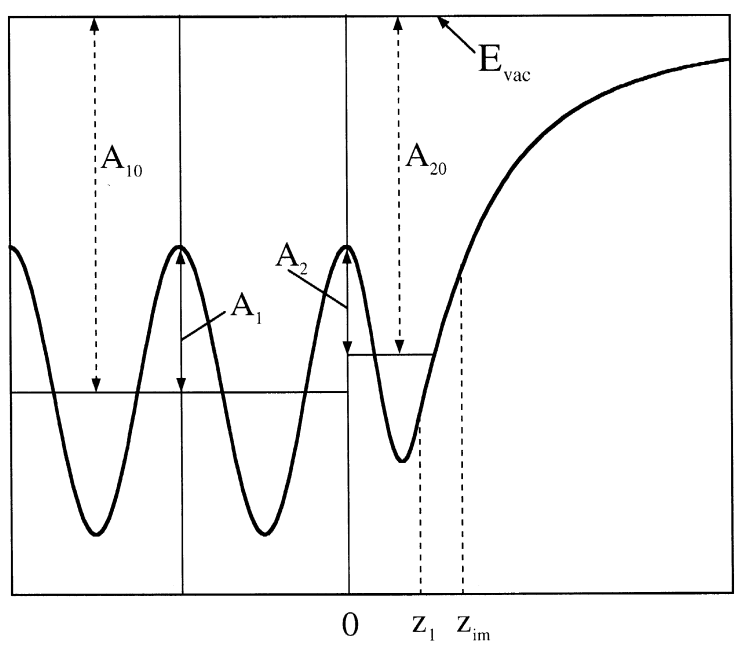

Fig. 13. Schematic plot of the model potential of Eq. (49). Vertical solid lines represent the position of atomic layers.

ples calculations. Probability amplitudes of the $n=1$ image state on $\mathrm{Li}(110)$, as obtained from either a self-consistent pseudopotential calculation or the one-dimensional model potential of Eq. (49) are represented in Fig. 14, showing that the agreement between the two curves is excellent. The probability amplitude of the $n=1$ image state on $\mathrm{Cu}(100)$, as obtained from the one-dimensional model potential of Eq. (49), also shows very good agreement with the result obtained with the use of a FLAPW calculation [136] (see Fig. 14b).

Assuming that corrugation effects, i.e., effects associated with spatial variations of the potential in the plane parallel to the surface, are not important and that the three-dimensional potential can be described by the $(x, y)$-plane average, one-electron wave functions and energies are taken to be given by Eqs. (20) and (21), respectively. Within a many-body self-energy formalism, the linewidth of the $n$ image state with energy $\varepsilon_{k, n}$ is then obtained from Eq. (22) or, within either the GW or the GW $\Gamma$ approximation, from Eq. (23).

\subsubsection{Results and discussion}

First of all, we present results obtained with use of the one-dimensional model potential (MP) of Eq. (49), and compare with experimental and other theoretical results. A summary of experimental results for image-state lifetimes in noble and transition metal surfaces, as obtained from either 2PPE or TR-2PPE measurements, is presented in Table 2, together with the result of theoretical calculations at the $\bar{\Gamma}$ point $\left(\boldsymbol{k}_{\|}=0\right)$. We note that there are large differences between 2PPE and TR-2PPE experimental results for copper and silver surfaces, the lifetime broadening derived from recent very-high resolution TR-2PPE measurements being smaller than that obtained from 2PPE experiments by nearly a factor of 2 .

Theoretical calculations presented in Table 2 can be classified into two groups. First, there is the heuristic approximation of Eqs. (47) and (48), which was carried out by Fauster and Steinmann [74] for a variety of metal surfaces. This approach results in a semiquantitative agreement with $2 \mathrm{PPE}$ measurements, except for the (111) surfaces of noble metals
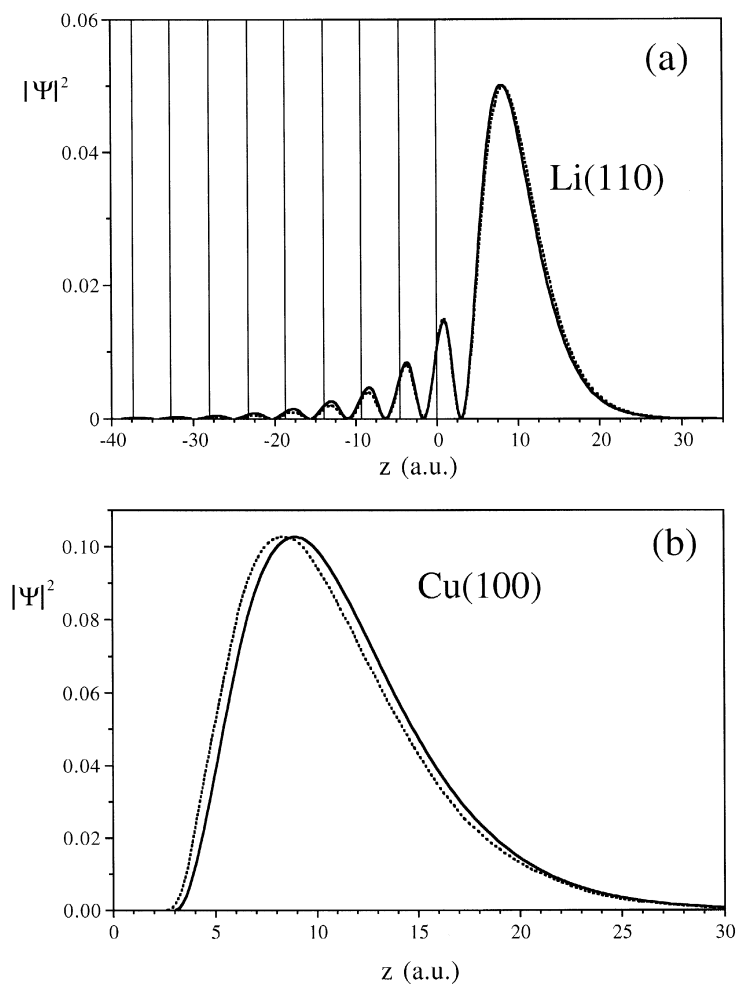

Fig. 14. The probability amplitude of the $n=1$ image state on (a) the (110) surface of $\mathrm{Li}$, as obtained from the model potential of Eq. (49) (solid line) and from pseudopotential calculations (dotted line), and (b) the (100) surface of $\mathrm{Cu}$, as obtained from the model potential of Eq. (49) (solid line) and from linear augmented plane-wave calculations (dotted line). Vertical solid lines represent the position of atomic layers. 
Table 2

Linewidth (inverse lifetime) of image states, in $\mathrm{meV}$

\begin{tabular}{|c|c|c|c|c|}
\hline Surface & Image state & 2PPE & TR2PPE & Theory \\
\hline$\overline{\mathrm{Cu}(100)}$ & $\begin{array}{l}n=1 \\
n=2 \\
n=3\end{array}$ & $28 \pm 6^{a}$ & $\begin{aligned} 16.5 & \pm 3 / 2^{\mathrm{b}, \mathrm{c}} \\
5.5 & \pm 0.8 / 0.6^{\mathrm{b}, \mathrm{c}} \\
2.20 & \pm 0.16 / 0.14^{\mathrm{b}, \mathrm{c}}\end{aligned}$ & $\begin{array}{l}18^{\mathrm{a}} ; 26^{\mathrm{d}} ; 22^{\mathrm{e}} \\
5^{\mathrm{e}} \\
1.8^{\mathrm{e}}\end{array}$ \\
\hline $\mathrm{Cu}(111)$ & $n=1$ & $16 \pm 4^{\mathrm{f}} ; 85 \pm 10^{\mathrm{a}, \mathrm{g}}$ & $38 \pm 14 / 9^{\mathrm{h}} ; 30^{\mathrm{i}}$ & $20^{\mathrm{j}} ; 421^{\mathrm{a}} ; 118^{\mathrm{d}} ; 38^{\mathrm{e}}$ \\
\hline $\operatorname{Ag}(100)$ & $\begin{array}{l}n=1 \\
n=2 \\
n=3\end{array}$ & $\begin{array}{l}21 \pm 4^{\mathrm{a}} \\
3.7 \pm 0.4^{\mathrm{a}}\end{array}$ & $\begin{array}{l}26 \pm 18 / 7^{\mathrm{k}} ; 12 \pm 1^{\mathrm{c}} \\
3.7 \pm 0.4^{\mathrm{k}} ; 4.1 \pm 0.3 / 0.2^{\mathrm{c}} \\
1.83 \pm 0.08^{\mathrm{c}}\end{array}$ & $\begin{array}{l}22^{\mathrm{a}} ; 25^{\mathrm{d}} \\
5^{\mathrm{d}}\end{array}$ \\
\hline $\operatorname{Ag}(111)$ & $n=1$ & $45 \pm 10^{\mathrm{a}} ; 55^{1}$ & $22 \pm 10 / 6^{\mathrm{m}}$ & $58^{\mathrm{j}} ; 123^{\mathrm{a}} ; 110^{\mathrm{d}}$ \\
\hline $\mathrm{Au}(111)$ & $n=1$ & $160 \pm 40^{\mathrm{a}}$ & & $617^{\mathrm{a}}$ \\
\hline $\operatorname{Pd}(111)$ & $n=1$ & $70 \pm 20^{\mathrm{a}}$ & & $40^{\mathrm{a}} ; 35^{\mathrm{n}}$ \\
\hline $\mathrm{Ni}(100)$ & $n=1$ & $70 \pm 8^{a}$ & & $24^{\mathrm{a}}$ \\
\hline $\mathrm{Ni}(111)$ & $n=1$ & $84 \pm 10^{\mathrm{a}}$ & & $40^{\mathrm{a}}$ \\
\hline $\operatorname{Co}(0001)$ & $n=1$ & $95 \pm 10^{\mathrm{a}}$ & & $40^{\mathrm{a}}$ \\
\hline $\mathrm{Fe}(110)$ & $n=1$ & $130 \pm 30^{\mathrm{a}}$ & & $95^{\mathrm{a}}$ \\
\hline
\end{tabular}

${ }^{a}$ Ref. [74], Th. Fauster and W. Steinmann.

${ }^{b}$ Ref. [33], U. Höfer et al.

${ }^{c}$ Ref. [35], I.L. Shumay et al.

${ }^{\mathrm{d}}$ Ref. [81], E.V. Chulkov et al.

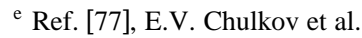

${ }^{\mathrm{f}}$ Ref. [125], S. Schuppler et al.

g Ref. [126], W. Wallauer and Th. Fauster.

${ }^{\mathrm{h}}$ Ref. [30], M. Wolf et al.

${ }^{i}$ Ref. [31], E. Knoesel et al., at low temperature, $T=25 \mathrm{~K}$.

${ }^{j}$ Ref. [66], P.L. de Andres et al.

${ }^{\mathrm{k}}$ Ref. [124], R.W. Schoenlein et al.

${ }^{1}$ Ref. [154], W. Merry et al.

${ }^{m}$ Ref. [34], J.D. McNeil et al.

${ }^{\mathrm{n}}$ Present work.

and the (100) surface of Ni. Similar computations were performed in Ref. [81] for the $n=1$ and $n=2$ image states on $\mathrm{Cu}$ and $\mathrm{Ag}$ surfaces, with use of the penetration of the image-state wave function that results from the one-dimensional model potential of Eq. (49). Though an accurate description of the penetration of the $n=1$ image-state wave function yields better agreement, in the case of $\mathrm{Cu}(111)$, with the experiment, this heuristic approach is still in semiquantitative agreement with TR-2PPE measurements. A quantitative agreement was found for the $n=2$ image-state linewidth in $\mathrm{Cu}(111)$.

In the other group of calculations the many-body self-energy formalism described in Section 3 was used for the evaluation of the lifetime of image states [77-80], resulting in a quantitative agreement with TR-2PPE measurements of the lifetime of image states on $\mathrm{Cu}$ surfaces and showing, therefore, that the present state of the theory enables to accurately predict the broadening of image states on metal surfaces.

To illustrate the importance that an accurate description of the self-energy might have on the evaluation of the linewidth, we show in Fig. 15 $\operatorname{Im}\left[-\Sigma\left(z, z^{\prime} ; \boldsymbol{k}_{\|}=0, E_{1}\right)\right]$ of the $n=1$ image-state electron at the $\bar{\Gamma}$ point $\left(\boldsymbol{k}_{\|}=0\right)$ on the (111) and (100) surfaces of $\mathrm{Cu}$. $\operatorname{Im}\left[-\Sigma\left(z, z^{\prime} ; \boldsymbol{k}_{\|}=0, E_{1}\right)\right]$ is represented in this figure as a function of $z$ for a fixed value of $z^{\prime}$ in the vacuum side of the surface (upper panel), within the bulk (middle panel), and at the surface (lower panel). It is obvious from this figure that the imaginary part of the self-energy is highly nonlocal [149], and strongly depends on the $z$ and $z^{\prime}$ coordinates. We note that $\operatorname{Im}\left[-\Sigma\left(z, z^{\prime} ; k_{\|}=0, E_{1}\right)\right]$ presents a maximum at $z=z^{\prime}$ when $z$ is located at the surface, and surface states can, therefore, play an important role in the decay mechanism of image states. The magnitude of 

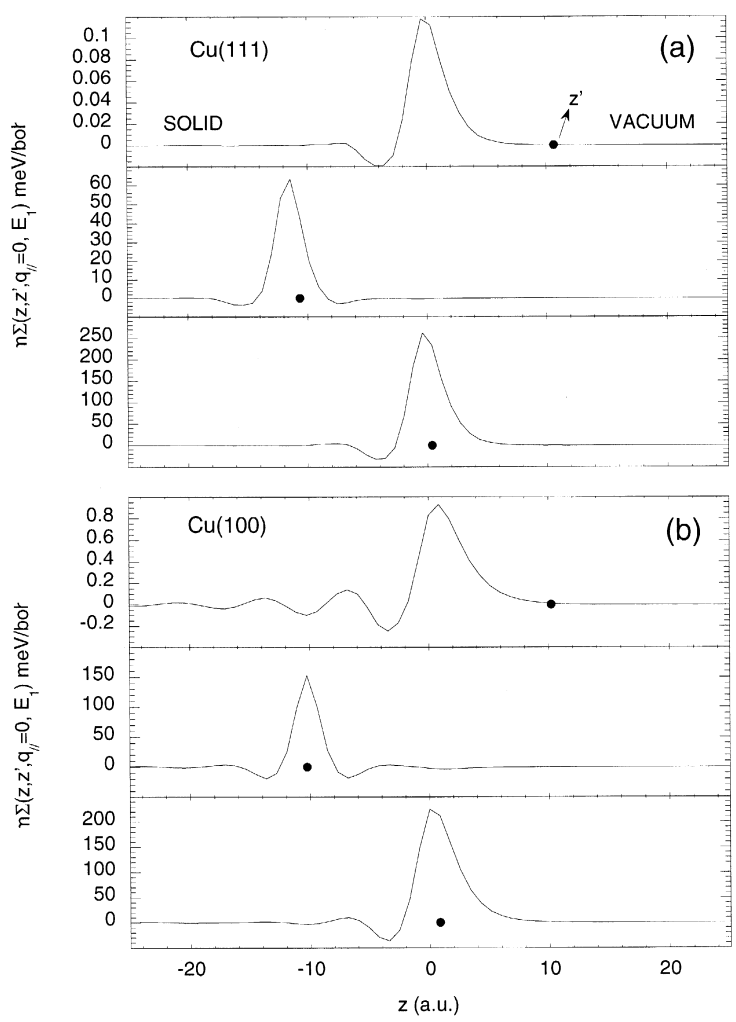

Fig. 15. Imaginary part of the electron self-energy, versus $z$, for three fixed values of $z^{\prime}$ (solid circles), as calculated for the $n=1$ image state on (a) $\mathrm{Cu}(111)$ and (b) $\mathrm{Cu}(100)$.

this maximum is plotted, as a function of $z^{\prime}$, in Fig. 16 , showing that it is an oscillating function of $z$ within the bulk ${ }^{8}$, and reaches its highest value at the surface.

It is interesting to note from Fig. 16a that the magnitude of $\operatorname{Im}\left[-\Sigma\left(z, z^{\prime} ; \boldsymbol{k}_{\|}=0, E_{1}\right)\right]$ is larger at the surface for $\mathrm{Cu}(111)$ than for $\mathrm{Cu}(100)$. Though the (100) surface of $\mathrm{Cu}$ only presents an intrinsic surface resonance, in the case of the (111) surface of $\mathrm{Cu}$ there is an intrinsic surface state just below the Fermi level. This intrinsic surface state provides a new channel for the decay of image states, thereby enhancing the imaginary part of the self-energy and the linewidth. The role that the intrinsic surface state

\footnotetext{
${ }^{8}$ The oscillatory behaviour within the bulk is dictated by the periodicity of the amplitude of final-state wave functions $\phi_{f}(z)$ in periodic crystals.
}

on $\mathrm{Cu}(111)$ plays in the decaying mechanism of image states is obvious from Fig. 16b, where contributions to the maximum self-energy coming from transitions to the intrinsic surface state and from transitions to bulk states have been plotted separately by dashed and dotted lines, respectively. The intrin-
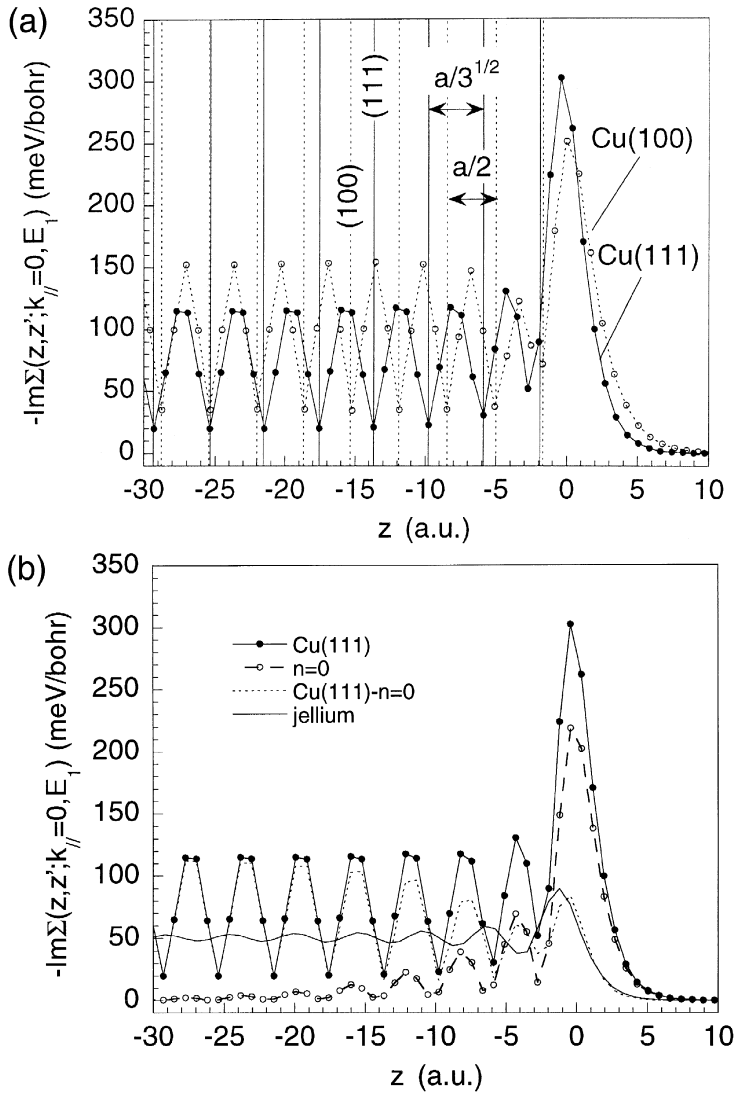

Fig. 16. (a) Maximum value of $\operatorname{Im}\left[-\Sigma\left(z, z^{\prime} ; \boldsymbol{k}_{\|}=0 ; E_{1}\right)\right]$ (see the text) for the $n=1$ image state on $\mathrm{Cu}(111)$ (solid line) and $\mathrm{Cu}(100)$ (dotted line). Vertical lines represent the position of atomic layers in $\mathrm{Cu}(111)$ (solid lines) and $\mathrm{Cu}(100)$ (dotted lines). (b) As in (a), for the separate contributions to the $n=1$ image state on $\mathrm{Cu}(111)$. The solid line with circles describes the total maximum value of $\operatorname{Im}\left[-\Sigma\left(z, z^{\prime} ; \boldsymbol{k}_{\|}=0 ; E_{1}\right)\right]$, the dashed line represents the contribution coming from the decay into the intrinsic surface state, and the dotted line, the contribution from the decay into bulk states. The solid line represents the result of replacing the realistic model-potential $\phi_{f}(z)$ final wave functions entering Eq. (23) by the self-consistent jellium LDA eigenfunctions of the one-electron Kohn-Sham hamiltonian but with the restriction that only final states with energy $\varepsilon_{f}$ lying below the projected band gap are allowed. 
Table 3

Calculated contributions to the linewidth, in $\mathrm{meV}$, of the $n=1$ image state on $\mathrm{Cu}$ surfaces

\begin{tabular}{lllll}
\hline Surface & $\Gamma_{\text {bulk }}$ & $\Gamma_{\text {vac }}$ & $\Gamma_{\text {inter }}$ & $\Gamma$ \\
\hline $\mathrm{Cu}(100)$ & 24 & 14 & -16 & 22 \\
$\mathrm{Cu}(111)$ & 44 & 47 & -54 & 37 \\
\hline
\end{tabular}

sic surface state provides a $\sim 75 \%$ of the decay mechanism at the surface. The intrinsic-surface-state contribution to the total linewidth of the $n=1$ image state on $\mathrm{Cu}(111)$ was found to be of about $40 \%$ $[77,78]$. Similarly, lower lying image states can give noticeable contributions to the linewidth of excited, i.e., $n=2,3, \ldots$ image states. For example, the decay from the $n=2$ to the $n=1$ image state on $\mathrm{Cu}(100)$ yields a linewidth of $0.5 \mathrm{meV}$, i.e. a $10 \%$ of the total $n=2$ image-state linewidth. The decay from the $n=3$ to the $n=1$ image state on $\mathrm{Cu}(100)$ yields a linewidth of $0.17 \mathrm{meV}$, and the decay from the $n=3$ to the $n=2$ image state on $\mathrm{Cu}(100)$ yields a linewidth of $0.05 \mathrm{meV}$, i.e. $\sim 10-15 \%$ of the total linewidth.

Coupling of image states with the crystal occurs through the penetration of the image-state wave function and, also, through the evanescent tails of bulk and surface states outside the crystal. To illustrate this point, the linewidth $\Gamma=\tau^{-1}$ can be split as follows

$\Gamma=\Gamma_{\text {bulk }}+\Gamma_{\text {vac }}+\Gamma_{\text {inter }}$,

where $\Gamma_{\text {bulk }}, \Gamma_{\text {vac }}$, and $\Gamma_{\text {inter }}$ represent bulk, vacuum and interface contributions, respectively. They are



Fig. 17. Schematical representation of the electronic structure of $\mathrm{Cu}(111)$ and $\mathrm{Cu}(100)$. 
Table 4

Linewidths $\Gamma_{1}$ and $\Gamma_{2}$, in $\mathrm{meV}$, of the $n=1$ image state on $\mathrm{Cu}(111)$, as calculated for non-zero momenta parallel to the surface and with use of two models for the $n=1$ image-state wave function (see text and Ref. [78])

\begin{tabular}{lll}
\hline$k(\mathrm{au})$ & $\Gamma_{1}$ & $\Gamma_{2}$ \\
\hline 0.0570 & 38.1 & 38.9 \\
0.0912 & 38.5 & 43.6 \\
0.1026 & 38.7 & 47.0 \\
\hline
\end{tabular}

obtained by confining the integrals in Eq. (23) to either bulk $\left(z<0, z^{\prime}<0\right)$, vacuum $\left(z>0, z^{\prime}>0\right)$, or vacuum-bulk $\left(z<0, z^{\prime}>0 ; z>0, z^{\prime}<0\right)$ coordinates. These separate contributions to the linewidth of image states on $\mathrm{Cu}$ surfaces are shown in Table 3. We note that the contribution to $\Gamma$ coming from the interference term $\Gamma_{\text {inter }}$ is comparable in magnitude and opposite in sign to both bulk and vacuum contributions. This is a consequence of the behaviour of the imaginary part of the two-dimensional Fourier transform of the self-energy, as discussed in Ref. [78]. The contributions $\Gamma_{\mathrm{vac}}$ and $\Gamma_{\text {inter }}$ almost completely compensate each other, and, in a first approximation, the total linewidth $\Gamma$ can be represented by the bulk contribution $\Gamma_{\text {bulk }}$ within an accuracy of $\sim 30 \%$.

The linewidth of image states can vary as a function of the two-dimensional momentum $\boldsymbol{k}_{\|}$. In Fig. 17 we show schematically the projection of the bulk band structure onto the (111) and (100) surfaces of $\mathrm{Cu}$. In the case of the (111) surface of $\mathrm{Cu}$, the $n=1$ image state becomes a resonance at $\left|\boldsymbol{k}_{\|}\right| \sim$ $0.114 \mathrm{a}_{0}^{-1}$, thereby the image-state wave function presenting a larger penetration into the bulk and an enhanced linewidth. Table 4 shows the linewidth of the $n=1$ image state on $\mathrm{Cu}(111)$, as obtained for three different values of $\boldsymbol{k}_{\|}$in the range $0-0.114$ $\mathrm{a}_{0}^{-1} \quad{ }^{9}$. In these calculations two approaches for the $n=1$ image-state wave function have been used. First, it has been obtained as an eigenfunction of the model potential of Eq. (49), with the parameters chosen so as to reproduce the width and position of

\footnotetext{
${ }^{9}$ The intraband contribution to the linewidth, coming from transitions between the states $\phi_{\boldsymbol{k}_{\|}, n=1}$ and $\phi_{\boldsymbol{k}_{\|}^{\prime}, n=1}$ with $\boldsymbol{k}_{\|} \neq \boldsymbol{k}_{\|}^{\prime}$ has not been included in this calculation.
}

the energy gap and the binding energies at the $\bar{\Gamma}$ point $\left(\boldsymbol{k}_{\|}=0\right)$. Secondly, the $n=1$ image-state wave function has been obtained with use of the model potential of Eq. (49), but with the parameters chosen so as to reproduce the width and position of the energy gap and the binding energies at the corresponding values of $\boldsymbol{k}_{\|}$, thus allowing for the penetration of the image-state wave function into the bulk to increase with $\boldsymbol{k}_{\|}$. Though both approximations yield an image-state linewidth that increases with $\boldsymbol{k}_{\|}$, it increases very slowly within the first approach and more rapidly within the second approach, showing the key role that the penetration of the image-state wave function plays in the decay mechanism, i.e., as the coupling of the image-state wave function with bulk and intrinsic-surface states increases, the image-state linewidth is enhanced.

As all theoretical calculations presented in Tables 2, 3 and 4 have been obtained with use of the wave functions of Eq. (20), surface corrugation has not been taken into account. Estimating the influence of surface corrugation on the image-state broadening requires the use of three-dimensional wave functions for the evaluation of initial and final states and, also, for the evaluation of the screened interaction. Work along these lines is now in progress.

An approximate way of including surface-corrugation effects on the final-state wave functions is to account for the actual effective mass of the intrinsic-surface and all bulk states, as obtained from the theoretically or experimentally determined dispersion of these states. In Table 5 we compare the

Table 5

Lifetimes of image states on $\mathrm{Cu}$ surfaces, in $\mathrm{fs}$

\begin{tabular}{llcl}
\hline Surface & $\begin{array}{l}\text { Image } \\
\text { state }\end{array}$ & TR2PPE & $\begin{array}{l}\text { Model potential } \\
\text { calculation }\end{array}$ \\
\hline $\mathrm{Cu}(100)$ & $n=1$ & $40 \pm 6^{\mathrm{a}, \mathrm{b}}$ & $30^{\mathrm{c}} ; 38^{\mathrm{d}}$ \\
& $n=2$ & $120 \pm 15^{\mathrm{a}, \mathrm{b}}$ & $132^{\mathrm{c}} ; 168^{\mathrm{d}}$ \\
& $n=3$ & $300 \pm 20^{\mathrm{a}, \mathrm{b}}$ & $367^{\mathrm{c}} ; 480^{\mathrm{d}}$ \\
$\mathrm{Cu}(111)$ & $n=1$ & $18 \pm 5^{\mathrm{e}} ; 22 \pm 5^{\mathrm{f}}$ & $17.5^{\mathrm{c}} ; 22.5^{\mathrm{d}, \mathrm{g}}$ \\
\hline
\end{tabular}

\footnotetext{
${ }^{a}$ Ref. [33], U. Höfer et al.

${ }^{\mathrm{b}}$ Ref. [35], I.L. Shumay et al.

${ }^{c}$ Ref. [77], E.V. Chulkov et al.

${ }^{\mathrm{d}}$ Ref. [80], I.Sarria et al.

${ }^{\mathrm{e}}$ Ref. [30], M. Wolf et al.

${ }^{\mathrm{f}}$ Ref. [31], E. Knoesel et al., at low temperature, $T=25 \mathrm{~K}$.

${ }^{g}$ Ref. [78], J. Osma et al.
} 
results of this calculation $[78,80]$ with recent accurate TR-2PPE measurements of the lifetime of image states on $\mathrm{Cu}$ surfaces, showing very good agreement between theory and experiment.

The present state of a theory that uses the self-energy formalism in combination with an accurate description, within a quasi one-dimensional model, of the key aspects of image states, namely, the position and width of the energy gap and the binding energies of the intrinsic and image-potential surface states has been shown to give quantitative account of the lifetime of image states on metal surfaces. Moreover, this theory also gives a linewidth of the Shockley surface state on $\mathrm{Cu}(111)$ [148] at the $\bar{\Gamma}$ point that is in excellent agreement with recent very-high-resolution angle-resolved photoemission measurements $[145,146]$. The calculated inelastic linewidth has been found to be of $26 \mathrm{meV}$, while measurements give 30 $\mathrm{meV}$ [145] and $21 \pm 5 \mathrm{meV}$ [146]. This calculation [148] emphasizes the extremely important role that the intrinsic surface state plays in the decaying mechanism of this state at the $\bar{\Gamma}$ point, resulting in a contribution of $\sim 70 \%$ of the total linewidth. This surface-state contribution explains the difference between the experimental data $[145,146]$ and theoretical results obtained within a bulk description of the broadening mechanism.

If dielectric layers are grown on the metal substrate, one can analyze the layer growth by simply looking at the energetics and lifetimes of image-state electrons [150]. Deposition of an overlayer on a metal substrate can change drastically the properties of image states, such as binding energies, wave functions, and lifetimes. This change depends on weather the adsorbate is a transition metal, an alkali metal, or a noble gas atom. In particular, deposition of alkali metal adlayer on $\mathrm{Cu}(111)$ decreases the work function by nearly a factor of 2 [151]. The linewidths of image states on a single layer of $\mathrm{Na}$ and $\mathrm{K}$ on $\mathrm{Cu}(111), \mathrm{Fe}(110)$, and $\mathrm{Co}(0001)$ were measured with 2PPE by Fisher et al. [152]. A large value of $150 \mathrm{meV}$ was obtained for the first image state, which is quite close to the linewidth of the $n=1$ image state on $\mathrm{Fe}(110)$ and $\mathrm{Co}(0001)$ but much larger than the linewidth of the $n=1$ image state on $\mathrm{Cu}(111)$ [74]. All these values were measured with 2PPE, and they include both energy and phase relaxation contributions. Additionally, these experiments showed the presence of the $n=0$ intrinsic surface state generated by the $\mathrm{Na} / \mathrm{K}$ layer, which replaces the intrinsic surface state on $\mathrm{Cu}(111)$. More accurate measurements of the intrinsic linewidth can be obtained with use of TR-2PPE spectroscopy. Nevertheless, the influence of impurities and imperfections on the linewidth remains to be evaluated. For more realistic estimates of the intrinsic linewidth, accurate models and/or first-principles calculations are necessary. The same applies to other metal overlayers [153].

Overlayers of $\mathrm{Xe}$ and $\mathrm{Kr}$ on $\mathrm{Ag}(111), \mathrm{Cu}(111)$, and $\mathrm{Ru}(0001)$ have been studied recently with use of 2PPE [154,155] and TR-2PPE [30,31,156-158] spectroscopies. All these measurements have shown that the lifetime of the $n=1$ state increases significantly upon deposition of the noble atom adlayer on all metal substrates of interest. Qualitatively, this increase can be explained by the fact that the interaction of the image-state electron with the closed Xe or $\mathrm{Kr}$ valence-shell is repulsive and, therefore, the probability amplitude of this state moves away from the crystal, as compared to the simple case of clean metal surfaces. Therefore, the coupling to the substrate decreases and the lifetime increases. The same qualitative argument can also explain the decrease of the binding energy of image states upon deposition of $\mathrm{Xe}$ or $\mathrm{Kr}$ on metal substrates. Moreover, Harris et al. [34,155] have studied the evolution of image states as a function of the number of deposited atomic layers of $\mathrm{Xe}$ on $\mathrm{Ag}(111)$. They have found that with increase of a number of Xe layers the $n=2$ and $n=3$ image states evolve into quantum-well states of the overlayer. A qualitative interpretation of this behaviour of image states has been given, within a macroscopic dielectric continuum model [155]. Unfortunately, no microscopic investigation of the image-state evolution of adlayers on metal surfaces that takes into account the band structure of both the substrate and the overlayer has been carried out.

Defects on the surface or adsorbed particles cause electron scattering processes that lead to phase relaxation of the wave function. This can be monitored in real time, in order to extract relevant information. In fact, measurements of the $n=1$ and $n=2$ imagepotential states of $\mathrm{CO}$ adsorbed in $\mathrm{Cu}(100)$ indicate a decreasing dephasing time when the $\mathrm{CO}$ molecules form an ordered $\mathrm{c}(2 \times 2)$ structure on the surface 
[144,147]. Furthermore, measurements on $\mathrm{Cu}(100)$ [144] have shown correlation between decay and dephasing, on the one hand, and the existence of surface defects, on the other hand. A first-principles description of this problem is still lacking, due to intrinsic difficulties in dealing with the loss of twodimensional translational symmetry.

Another important field of research is the understanding of the processes leading to the electronic relaxation in magnetic materials. The spin-split image states on magnetic surfaces [159-162] offer the possibility of extracting information about the underlying surface magnetism. These spin-split states can decay in different ways and, therefore, their linewidths can be different. In particular, spin-resolved inverse photoemission experiments on $\mathrm{Fe}(110)$ [160] give an intrinsic linewidth of 140 (70) $\mathrm{meV}$ for the first minority (majority) image state. The difference in the lifetime is of the order of the total linewidth of the $n=1$ image state on other metal surfaces. At the same time, as the spin-splitting is only of $\sim 8 \%$ of the total binding energy ( $E_{1}=$ $-0.73 \mathrm{eV})$, it is unlikely that this splitting is responsible for the large difference between linewidths. Hence, one has to resort to details of the phase space of final states and to the screened Coulomb interaction as responsible for this effect. Work along these lines is now in progress.

All these problems are of technological relevance and pose technical and theoretical questions that need to be answered in order to make a correct interpretation of what is really being measured. One technique is based on the ab initio description of the fast-dynamics of a wave-packet of excited electrons in front of the surface [163]. The time evolution will pick up all the relevant information concerning scattering processes and electronic excitations that can be mapped directly with experiments [33]. On a more complex and fundamental level, there is the theoretical description of coupled electron-ion dynamics, which is relevant in many experiments.

\section{Future}

We present here a brief summary of on-going and future work in the field of inelastic electron scattering in solids and, in particular, in the investigation of electron and hole inelastic lifetimes in bulk materials and low-dimensional structures. The advance in our knowledge is closely linked to the experimental developments that combine state-of-the-art angle-resolved 2PPE with ultrafast laser technology. These investigations might be relevant for potential technological applications, such as the control of chemical reactions in surfaces and the developing of new materials for opto-electronic devices.

A theoretical and experimental challenge is the description of the reactivity at surfaces. Experiments are being performed nowadays directed to get a deeper understanding of the electronic processes involved. We note that electronic excitation is the initial step in a chemical reaction, and the energetics and lifetimes of these processes directly govern the reaction probability. For example, we can achieve chemical selectivity through a femtosecond activation of the chemical reaction [164]. This shows clearly that nonrandom dissociation exists in polyatomic molecules on the femtosecond time-scale, by exciting the reactant to high energies (well above the threshold for dissociation) and sampling the products on time scales that are shorter than the rate for intramolecular vibrational energy-distribution (this concept is relevant in chemical reactivity and assumes ergodicity or, equivalently, that the internal energy is statistically redistributed). The idea of ergodicity has to be revisited in this short time-scale.

Very recently, it has been shown that selective adsorption of low-energy electrons into an imagepotential state, followed by inelastic scattering and desorption, can provide information on the interaction between these states and the substrate [165]. A deep theoretical analysis of this interaction, as well as the role of the substrate/adsorbate band structure, is still lacking and is needed in order to interpret the experimental data.

So far, we have concentrated our attention to the investigation of bulk and surfaces, i.e., extended systems. From a technological point of view, and due to the fast miniaturization of the magneto- and opto-electronic components in current devices, the study of the electron dynamics in nanostructures is of relevance. For example, alkali metals that have image states as resonances would have, in a finite piece of material, a well-defined image state with a long lifetime. These states are spatially located out- 
side the nanostructure and, at least in principle, could be used in a possible self-assembling mechanism to build controlled structures made of clusters, and also as an efficient external probe for chemical characterization. Measurements on negatively charged clusters would be able to assess this effect, as well as its size dependence. Experiments performed on a $\mathrm{Na}_{91}^{-}$cluster have looked at two decay mechanisms for the collective excitation, namely, electron and atom emission. The estimated electronic escape time is of the order of $1 \mathrm{fs}$ [166]. The relaxation time for two-electron collisions in small sodium clusters has been estimated theoretically at the level of a time-dependent local-density-functional approach (TDLDA) [167]. The computed values are in the range of 3-50 fs, which are between the direct electron emission and the ionic motion ( $>100 \mathrm{fs}$ ). These values compete with the scale for Landau damping (coupling of the collective excitation to neighbouring particle-hole states). A first non-perturbative approach to the quasiparticle lifetime in a quantum dot has been presented in Ref. [168], where localized (quasiparticle states are single-particle-like states) and delocalized (superposition of states) regimes are identified. Furthermore, if we wish to use these nanostructures in devices, we need to understand the scattering mechanism that controls the electronic transport at the nanoscale level. We expect new physical phenomena to appear in detailed time-resolved experiments in these systems, related to quantum confinement. In summary, the investigation of electronelectron interactions in nanostructures is still in its infancy, and much work is expected to be done in the near future. In particular, we are planning to investigate electron lifetimes in fullerene-based materials, such as $\mathrm{C}_{60}$ and carbon nanotubes.

Asymmetries in electron lifetimes arise from the different nature and localization of electrons; in this sense, noble and transition metals offer a valuable framework to deal with different type of electrons that present various degrees of localization. New theoretical techniques should be able to address the calculation of excitations and inelastic electron lifetimes, including to some extent electron-phonon couplings [which might be important and even dominant for high enough temperatures and very-low-energy electrons] and also both impurity and grain-boundary scattering. Final-state effects have been neglected in most practical implementations, and they might be important when there is strong localization, as in the case of transition and rare earth materials. In the case of semiconductors, electron-hole interactions (excitonic renormalization) strongly modify the singleparticle optical absorption profile, and they need to be included in the electronic response [169]. Although similar interactions are expected to be present in metals, the large screening in these systems makes their contribution less striking as compared to the case of semiconductors. However, in the case of low-dimensional structures they might play an important role in the broadening mechanism of excited electrons and holes.

All calculations presented in Sections 4 and 5 stop at the first iteration of the GW approximation. Although going beyond this approximation is possible, this has to be done with great care, since higher-order corrections tend to cancel out the effects of selfconsistency [170-172] (see Appendix B). As we start from an RPA-like screening, the net effect of including the so-called vertex corrections for screening electrons would be a reduction of the screening. Furthermore, a simpler and important effect to be included in the present calculations is related to the renormalization of the excitation spectral weight due to changes in the self-energy close to the Fermi surface. We know that this renormalization could be as large as 0.5 for $\mathrm{Ni}[173,174]$ and of the order of 0.8 for $\mathrm{Si}$ [175]. This modifies the energy of the excitation and, therefore, the lifetime. We aim to include such effects in the calculation of the inelastic electronic scattering process in noble and transition metals [176], along the lines described in the Appendix $\mathrm{B}$. The main idea is to work directly with the Green function in an imaginary-time/energy representation. The choice of representing the time/energy dependence on the imaginary rather than the real axis allows us to deal with smooth, decaying quantities, which give faster convergence. Only after the full imaginary-energy dependence of the expectation values of the self-energy operator has been established do we use a fitted model function (whose sophistication may be increased as necessary with negligible expense), which we then analytically continue to the real energy axis in order to compute excitation spectra and lifetimes [177-180]. Furthermore, this technique is directly connected with 
finite-temperature many-body Green functions, and can be used to directly address temperature effects on the lifetime that can be measured experimentally.

An interesting aspect in the theory of inelastic electron scattering appears when one looks at the energy dependence of the electron lifetime in layered materials. In a semimetal as graphite, the lifetime has been found to be inversely proportional to the energy above the Fermi level [181], in contrast to the quadratic behaviour predicted for metals with the use of Fermi-liquid theory (see Section 4). This behaviour has been interpreted in terms of electronplasmon interaction in a layered electron gas [181]; however, this is not consistent with the fact that a layered Fermi-liquid shows conventional electron lifetimes [182]. A different interpretation based on the particular band structure of graphite (with a nearly point-like Fermi surface) yielding a reduction of the screening can explain the linear dependence of the lifetime [183]. A similar linear dependence of the inelastic lifetime has been found for other semiconducting-layered compounds as $\mathrm{SnS}_{2}$ [184]. We are presently working on the evaluation, within the GW approximation, of electron lifetimes in these layered compounds [185]. The special band structure of graphite has also been invoked as responsible for the peculiar plasmon dispersion and damping of the surface plasmon [186]. Therefore, a careful analysis of the layer-layer interaction and broadening of the Fermi surface needs to be included, in order to understand this behaviour. We note that in a metal like Ni the imaginary part of the self-energy shows a quadratic Fermi-liquid behaviour, which becomes linear very quickly $[173,174]$.

Together with the self-energy approach discussed in this review, an alternative way of computing the excitation spectra of a many-body system, which is based on information gleaned from an ordinary ground-state calculation, is the time-dependent density-functional theory (TDDFT) [187-190]. In this approach, one studies how the system behaves under an external perturbation. The response of the system is directly related to the $N$-particle excited states of an $N$-particle system, in a similar manner as the one-particle Green function is related to the $(N+1)$ and $(N-1)$-particle excited states of the same system. TDDFT is an ideal tool for studying the dynamics of many-particle systems, which is based on a complete representation of the $\mathrm{XC}$ kernel, $K^{\mathrm{xc}}$, in time and space. One computes the time-evolution of the system [191-194] without resorting to perturbation theory and dealing, therefore, with an external field of arbitrary strength. The fact that the evolution of the wave function is mapped for a given time-interval helps one to extract useful information on the dynamics and electron relaxation of many-electron systems. The method does not stop on the linear response and includes, in principle, higher-order nonlinear response as well as multiple absorption and emission processes.

On a more pure theoretical framework, the connection between TDDFT and many-body perturbation theory is needed, in order to get further insight into the form of the frequency-dependent and non-local XC kernel $K^{\mathrm{xc}}$. If one were able to design an XC kernel that works for excitations as the LDA does for ground-state properties, then one could handle many interesting problems that are related to electron dynamics of many-electron systems.

In summary, many theoretical and experimental challenges related to the investigation of lifetimes of low-energy electrons in metals and semiconductors are open, and even more striking theoretical and experimental advances are ready to come in the near future. Lifetime measurements can be complementary to current spectroscopies for the attainment of information about general properties (structural, electronic, dynamical, ....) of a given system.

\section{Acknowledgements}

The authors would like to thank I. Campillo, M. A. Cazalilla, J. Osma, I. Sarria, V. M. Silkin, and E. Zarate, for their contributions to some of the results that are reported here, and M. Aeschlimann, Th. Fauster, U. Höfer, and M. Wolf, for enjoyable discussions. Partial support by the University of the Basque Country, the Basque Unibertsitate eta Ikerketa Saila, the Spanish Ministerio de Educación y Cultura, and Iberdrola S. A. is gratefully acknowledged.

\section{Appendix A. Linear response}

Take a system of interacting electrons exposed to an external potential $V^{\text {ext }}(\boldsymbol{r}, \omega)$. According to time- 
dependent perturbation theory and keeping only terms of first order in the external perturbation $V^{\operatorname{ext}}(\boldsymbol{r}, \omega)$, the charge density induced in the electronic system is found to be

$\rho^{\text {ind }}(\boldsymbol{r}, \omega)=\int \mathrm{d} \boldsymbol{r}^{\prime} \chi\left(\boldsymbol{r}, \boldsymbol{r}^{\prime} ; \omega\right) V^{\mathrm{ext}}\left(\boldsymbol{r}^{\prime}, \omega\right)$,

where $\chi\left(\boldsymbol{r}, \boldsymbol{r}^{\prime} ; \omega\right)$ represents the so-called linear density response function

$$
\begin{aligned}
\chi\left(\boldsymbol{r}, \boldsymbol{r}^{\prime}, \omega\right)= & \sum_{n} \rho_{n 0}^{*}(\boldsymbol{r}) \rho_{n 0}\left(\boldsymbol{r}^{\prime}\right) \\
& \times\left[\frac{1}{\omega-\omega_{n 0}+\mathrm{i} \eta}-\frac{1}{\omega+\omega_{n 0}+\mathrm{i} \eta}\right] .
\end{aligned}
$$

Here, $\omega_{n 0}=E_{n}-E_{0}$ and $\rho_{n 0}(\boldsymbol{r})$ represent matrix elements taken between the unperturbed many-particle ground state $\left|\Psi_{0}\right\rangle$ of energy $E_{0}$ and the unperturbed many-particle excited state $\left|\Psi_{n}\right\rangle$ of energy $E_{n}$ :

$\rho_{n 0}(\boldsymbol{r})=\left\langle\Psi_{n}|\rho(\boldsymbol{r})| \Psi_{0}\right\rangle$,

$\rho(\boldsymbol{r})$ being the charge-density operator,

$\rho(\boldsymbol{r})=-\sum_{i=1}^{N} \delta\left(\boldsymbol{r}-\boldsymbol{r}_{i}\right)$,

and $\boldsymbol{r}_{i}$ describing electron coordinates.

In a time-dependent Hartree or random-phase approximation, the electron density induced by the external potential, $V^{\operatorname{ext}}(\boldsymbol{r}, \omega)$, is approximated by the electron density induced in a noninteracting electron gas by the total field $V^{\text {ext }}(\boldsymbol{r}, \omega)+V^{\text {ind }}(\boldsymbol{r}, \omega)$ :

$$
\begin{aligned}
\rho^{\text {ind }}(\boldsymbol{r}, \omega)= & \int \mathrm{d} \boldsymbol{r}^{\prime} \chi\left(\boldsymbol{r}, \boldsymbol{r}^{\prime} ; \omega\right) \\
& \times\left[V^{\mathrm{ext}}\left(\boldsymbol{r}^{\prime}, \omega\right)+V^{\text {ind }}\left(\boldsymbol{r}^{\prime}, \omega\right)\right] .
\end{aligned}
$$

This approximation for the induced electron density can be written in the form of Eq. (A.1), with

$$
\begin{aligned}
\chi^{\mathrm{RPA}}\left(\boldsymbol{r}, \boldsymbol{r}^{\prime} ; \omega\right) \\
=\chi^{0}\left(\boldsymbol{r}, \boldsymbol{r}^{\prime} ; \omega\right)+\int \mathrm{d} \boldsymbol{r}_{1} \int \mathrm{d} \boldsymbol{r}_{2} \chi^{0}\left(\boldsymbol{r}, \boldsymbol{r}_{1} ; \omega\right) \\
\quad \times v\left(\boldsymbol{r}_{1}-\boldsymbol{r}_{2}\right) \chi^{\mathrm{RPA}}\left(\boldsymbol{r}_{2}, \boldsymbol{r}^{\prime} ; \omega\right),
\end{aligned}
$$

where $\chi^{0}\left(\boldsymbol{r}, \boldsymbol{r}^{\prime} ; \omega\right)$ is the density-response function of noninteracting electrons,

$$
\begin{aligned}
\chi^{0}\left(\boldsymbol{r}, \boldsymbol{r}^{\prime} ; \omega\right)= & 2 \sum_{i, j} \frac{\theta\left(E_{\mathrm{F}}-\omega_{i}\right)-\theta\left(E_{\mathrm{F}}-\omega_{j}\right)}{\varepsilon_{i}-\varepsilon_{j}+(\omega+\mathrm{i} \eta)} \\
& \times \phi_{i}(\boldsymbol{r}) \phi_{j}^{*}(\boldsymbol{r}) \phi_{j}\left(\boldsymbol{r}^{\prime}\right) \phi_{i}^{*}\left(\boldsymbol{r}^{\prime}\right),
\end{aligned}
$$

$\phi_{i}(\boldsymbol{r})$ representing a set of single-particle states of energy $\varepsilon_{i}$.

In the framework of time-dependent density-functional theory [187-190], the theorems of which generalize those of the usual density-functional theory $[114,115]$, the density-response function satisfies the integral equation

$$
\begin{aligned}
& \chi\left(\boldsymbol{r}, \boldsymbol{r}^{\prime} ; \omega\right) \\
& =\chi^{0}\left(\boldsymbol{r}, \boldsymbol{r}^{\prime} ; \omega\right)+\int \mathrm{d} \boldsymbol{r}_{1} \int \mathrm{d} \boldsymbol{r}_{2} \chi^{0}\left(\boldsymbol{r}, \boldsymbol{r}_{1} ; \omega\right) \\
& \quad \times\left[v\left(\boldsymbol{r}_{1}-\boldsymbol{r}_{2}\right)+K^{\mathrm{xc}}\left(\boldsymbol{r}_{1}, \boldsymbol{r}_{2} ; \omega\right)\right] \chi\left(\boldsymbol{r}_{2}, \boldsymbol{r}^{\prime} ; \omega\right),
\end{aligned}
$$

the kernel $K^{\mathrm{xc}}\left(\boldsymbol{r}, \boldsymbol{r}^{\prime} ; \omega\right)$ representing the reduction in the e-e interaction due to the existence of short-range XC effects. In the static limit $(\omega \rightarrow 0)$, DFT shows that [190]

$$
K^{\mathrm{xc}}\left(\boldsymbol{r}, \boldsymbol{r}^{\prime} ; \omega \rightarrow 0\right)=\left[\frac{\delta^{2} E_{x c}[n]}{\delta n(\boldsymbol{r}) \delta n\left(\boldsymbol{r}^{\prime}\right)}\right]_{n_{0}(\boldsymbol{r})},
$$

where $E_{x c}[n]$ represents the XC energy functional and $n_{0}(\boldsymbol{r})$ is the actual density of the electron system.

In the case of a homogeneous electron gas, one introduces Fourier transforms and writes

$\rho_{q, \omega}^{\text {ind }}=\chi_{q, \omega} V_{q, \omega}^{\text {ext }}$.

Within RPA,

$\chi_{\boldsymbol{q}, \omega}^{\mathrm{RPA}}=\chi_{\boldsymbol{q}, \omega}^{0}+\chi_{\boldsymbol{q}, \omega}^{0} v_{\boldsymbol{q}} \chi_{\boldsymbol{q}, \omega}^{\mathrm{RPA}}$,

where

$$
\begin{aligned}
\chi_{q, \omega}^{0}= & \frac{2}{\Omega} \sum_{k} n_{k}\left(1-n_{k+q}\right)\left[\frac{1}{\omega+\varepsilon_{k}-\varepsilon_{k+q}+\mathrm{i} \eta}\right. \\
& \left.-\frac{1}{\omega+\varepsilon_{k+q}-\varepsilon_{k}+\mathrm{i} \eta}\right],
\end{aligned}
$$


$v_{q}=4 \pi / q^{2}$ is the Fourier transform of the Coulomb potential, $\varepsilon_{\boldsymbol{k}}=\boldsymbol{k}^{2} / 2$, and $n_{\boldsymbol{k}}$ are occupation numbers, as given by Eq. (6).

In the more general scenario of TDDFT,

$$
\begin{aligned}
\chi_{\boldsymbol{q}, \omega} & =\chi_{\boldsymbol{q}, \omega}^{0}+\chi_{\boldsymbol{q}, \omega}^{0}\left(v_{\boldsymbol{q}}+K_{\boldsymbol{q}, \omega}^{\mathrm{xc}}\right) \chi_{\boldsymbol{q}, \omega} \\
& =\chi_{\boldsymbol{q}, \omega}^{0}+\chi_{\boldsymbol{q}, \omega}^{0} v_{\boldsymbol{q}}\left(1-G_{\boldsymbol{q}, \omega}\right) \chi_{\boldsymbol{q}, \omega},
\end{aligned}
$$

where $K_{q, \omega}^{\mathrm{xc}}$ is the Fourier transform of the $\mathrm{XC}$ kernel $K^{\mathrm{xc}}\left(\boldsymbol{r}, \boldsymbol{r}^{\prime}, \omega\right)$. In Eq. (A.13), we have set

$K_{\boldsymbol{q}, \omega}^{\mathrm{xc}}=-v_{\boldsymbol{q}} G_{\boldsymbol{q}, \omega}$,

$G_{q, \omega}$ being the so-called local-field factor. In the local-density approximation, which is rigorous in the long-wavelength limit $(q \rightarrow 0)$, it follows from Eqs. (A.9) and (A.14) that

$G_{q, 0}^{\mathrm{LDA}}=A\left(\frac{q}{q_{\mathrm{F}}}\right)^{2}$,

where

$A=\frac{1}{4}-\frac{4 \pi}{q_{\mathrm{F}}^{2}} \frac{\mathrm{d}^{2} E_{\mathrm{c}}}{\mathrm{d} n_{0}^{2}}$,

$E_{\mathrm{c}}\left(n_{0}\right)$ being the correlation contribution to the ground-state energy of the uniform electron gas. This quantity has been extensively studied, ranging from the simple Wigner interpolation formula $[95,195]$ to accurate parametrizations [196,197] based on Monte Carlo calculations by Ceperley and Alder [198].

Diffusion Monte Carlo calculations of the static density-response function of the uniform electron gas $[199,200]$ have shown that the LDA static local-field factor of Eq. (A.15) correctly reproduces the static response for all $q \leq 2 q_{\mathrm{F}}$, as long as the exact correlation energy is used to calculate $A$. For larger values of $q$ both exact and RPA density-response functions decay as $1 / q^{2}$, their difference being of order $1 / q^{4}$, and fine details of $G_{q, 0}$ are expected to be of little importance [201-203].

Calculations of the frequency dependence of the local-field factor were carried out by Brosens and coworkers [204] and, more recently, by Richardson and Ashcroft [205]. Local-field factors are in general expected to be complex for nonzero frequencies, and the importance of their frequency dependence is reflected, e.g., in the finite lifetime of the volume plasmon. For aluminum, measurements of $G_{q, \omega}$ have shown [206] a weak $\omega$ dependence of the local-field factor for energies below $\sim 35 \mathrm{eV}$.

If the homogeneous electron gas is exposed to an external test charge of density $\rho^{\operatorname{ext}}(\boldsymbol{r}, t)$, one writes

$V_{q, \omega}^{\mathrm{ext}}=v_{\boldsymbol{q}} \rho_{\boldsymbol{q}, \omega}^{\mathrm{ext}}$,

and with the aid of Eq. (A.10) one finds the Fourier transform of the total change of the charge density $\rho^{\text {tot }}(\boldsymbol{r}, t)=\rho^{\text {ext }}(\boldsymbol{r}, t)+\rho^{\text {ind }}(\boldsymbol{r}, t)$ to be given by the following expression:

$\rho_{q, \omega}^{\mathrm{tot}}=\epsilon_{q, \omega}^{-1} \rho_{q, \omega}^{\mathrm{ext}}$,

where $\epsilon_{q, \omega}$ is the so-called test-charge-test-charge dielectric function:

$\epsilon_{q, \omega}^{-1}=1+v_{q} \chi_{q, \omega}$.

Hence, this dielectric function screens the potential both generated and 'felt' by a test charge.

If the external potential is that generated by an electron, then one writes

$V_{q, \omega}^{\mathrm{ext}}=v_{\boldsymbol{q}}\left(1-G_{\boldsymbol{q}, \omega}\right) \rho_{\boldsymbol{q}, \omega}^{\mathrm{ext}}$,

and with the aid of Eq. (A.10) one finds Eq. (A.18), but now $\epsilon_{q, \omega}$ being the test-charge-electron dielectric function [99]:

$\epsilon_{q, \omega}^{-1}=1+v_{q}\left(1-G_{q, \omega}\right) \chi_{q, \omega}$.

By combining Eq. (A.13) with either Eq. (A.19) or Eq. (A.21), one can easily obtain the following expressions for the test-charge-test-charge and the test-charge-electron dielectric functions,

$\epsilon_{q, \omega}=1-\frac{v_{\boldsymbol{q}} \chi_{\boldsymbol{q}, \omega}^{0}}{1+v_{\boldsymbol{q}} G_{\boldsymbol{q}, \omega} \chi_{\boldsymbol{q}, \omega}^{0}}$

and

$\epsilon_{\boldsymbol{q}, \omega}^{-1}=1+v_{\boldsymbol{q}}\left(1-G_{\boldsymbol{q}, \omega}\right) \chi_{\boldsymbol{q}, \omega}$,

respectively. If the local-field factor $G_{q, \omega}$ is set equal to zero, both Eqs. (A.23) and (A.24) yield the RPA dielectric function $[94,95]$

$\epsilon_{\boldsymbol{q}, \omega}^{\mathrm{RPA}}=1-v_{\boldsymbol{q}} \chi_{\boldsymbol{q}, \omega}^{0}$.

In terms of $\epsilon_{q, \omega}^{\mathrm{RPA}}$, Eqs. (A.23) and (A.24) yield Eqs. (12) and (13), respectively. 


\section{Appendix B. GW approximation and beyond}

Let us introduce the many-body Green function [207]:

$$
\begin{aligned}
& \mathscr{G}\left(\boldsymbol{r} t, \boldsymbol{r}^{\prime} t^{\prime}\right) \\
& =-\mathrm{i} \theta\left(t-t^{\prime}\right)\left\langle\Psi_{0}^{N}\left|\psi(\boldsymbol{r}, t) \psi^{\dagger}\left(\boldsymbol{r}^{\prime}, t^{\prime}\right)\right| \Psi_{0}^{N}\right\rangle \\
& \quad+\mathrm{i} \theta\left(t^{\prime}-t\right)\left\langle\Psi_{0}^{N}\left|\psi^{\dagger}\left(\boldsymbol{r}^{\prime}, t^{\prime}\right) \psi(\boldsymbol{r}, t)\right| \Psi_{0}^{N}\right\rangle .
\end{aligned}
$$

In this equation, $\psi^{\dagger}(\boldsymbol{r}, t)\left|\Psi_{0}^{N}\right\rangle$ stands for a $(N+1)$ electron state in which an electron has been added to the system at point $\boldsymbol{r}$ and time $t$. When $t^{\prime}<t$, the many-body Green function gives the probability amplitude to detect an electron at point $\boldsymbol{r}$ and time $t$ when a (possibly different) electron has been added to the system at point $\boldsymbol{r}^{\prime}$ and time $t^{\prime}$. When $t^{\prime}>t$, the Green function describes the propagation of a many-body state in which one electron has been removed at point $\boldsymbol{r}$ and time $t$, that is, the propagation of a hole.

For a system of interacting electrons, there is little hope of calculating $\mathscr{G}\left(\boldsymbol{r}, \boldsymbol{r}^{\prime}, \omega\right)$ exactly. One usually has to resort to perturbation theory, starting from a suitably chosen one-electron problem with a Hamiltonian $H_{0}(\boldsymbol{r})$, eigenfunctions $\phi_{i}(\boldsymbol{r})$, and eigenenergies $\varepsilon_{i}$. Hence, the noninteracting Green function $\mathscr{G}^{0}\left(\boldsymbol{r}, \boldsymbol{r}^{\prime}, \omega\right)$ is given by the following expression [100]:

$\mathscr{G}^{0}\left(\boldsymbol{r}, \boldsymbol{r}^{\prime}, \omega\right)=\sum_{i} \frac{\phi_{i}^{*}(\boldsymbol{r}) \phi_{i}\left(\boldsymbol{r}^{\prime}\right)}{\omega-\varepsilon_{i}+\mathrm{i} \eta \operatorname{sgn}\left(\varepsilon_{i}-E_{\mathrm{F}}\right)}$.

In usual practice the LDA [115] is considered, which provides a local one-electron potential, $u_{\mathrm{LDA}}(\boldsymbol{r})$.

The exact Green function obeys the following Dyson's equation [100],

$$
\begin{aligned}
(\omega & \left.+\frac{1}{2} \nabla_{\boldsymbol{r}}^{2}-u(\boldsymbol{r})\right) \mathscr{G}\left(\boldsymbol{r}, \boldsymbol{r}^{\prime}, \omega\right) \\
& +\int \mathrm{d} \boldsymbol{r}^{\prime \prime} \Sigma\left(\boldsymbol{r}, \boldsymbol{r}^{\prime \prime}, \omega\right) \mathscr{G}\left(\boldsymbol{r}^{\prime \prime}, \boldsymbol{r}, \omega\right) \\
& =\boldsymbol{\delta}\left(\boldsymbol{r}-\boldsymbol{r}^{\prime}\right),
\end{aligned}
$$

where the integral kernel $\Sigma\left(\boldsymbol{r}, \boldsymbol{r}^{\prime}, \omega\right)$ is known as the self-energy. It can be understood as the complex non-local energy-dependent potential felt by the elec- tron added to the system at point $\boldsymbol{r}^{\prime}$ and time $t^{\prime}$. This potential arises from the response of the rest of electrons to the presence of the additional electron. However, one must be careful with this interpretation, since the many-body Green function for $t^{\prime}<t$ not only describes the propagation of the additional electron, but also that of the whole $(N+1)$-electron system. This means, for instance, that the self-energy also accounts for the exchange processes that occur in a system of indistinguishable particles.

To obtain the inelastic lifetime of one-electron-like excitations, called quasiparticles, one seeks for the poles of the many body Green function. A good estimate of the position of these poles can be obtained by projecting Eq. (B.3) onto the chosen basis of one-electron orbitals, and neglecting the off-diagonal terms in the self-energy, i.e.,

$\omega-\varepsilon_{i}-\Delta \Sigma_{i i}(\omega) \approx 0$,

where

$$
\begin{aligned}
\Delta \Sigma_{i i}(\omega)= & \int \mathrm{d} \boldsymbol{r} \int \mathrm{d} \boldsymbol{r}^{\prime} \phi_{i}^{*}(\boldsymbol{r})\left[\Sigma\left(\boldsymbol{r}, \boldsymbol{r}^{\prime} ; \omega\right)\right. \\
& \left.-u_{\mathrm{LDA}}(\boldsymbol{r}) \boldsymbol{\delta}\left(\boldsymbol{r}-\boldsymbol{r}^{\prime}\right)\right] \phi_{i}\left(\boldsymbol{r}^{\prime}\right) .
\end{aligned}
$$

In general, Eq. (B.4) has complex solutions at $\omega=E$ $-\mathrm{i} \Gamma / 2$, where $E$ is the excitation energy and $\Gamma$ the linewidth of the quasiparticle. Near the energyshell ( $\omega \sim \varepsilon_{i}, \varepsilon_{i}$ being the free-particle energy), one finds for the linewidth $\tau^{-1}=\Gamma$ :

$\tau^{-1}=-2 Z_{i} \operatorname{Im} \Delta \Sigma_{i i}\left(\varepsilon_{i}\right)$,

where

$Z_{i}=\left[1-\left.\frac{\partial \operatorname{Re} \Delta \Sigma_{i i}(\omega)}{\partial \omega}\right|_{\omega=\varepsilon_{i}}\right]^{-1}$

is a renormalization constant. On the energy-shell $\left(Z_{i} \sim 1\right)$, one writes

$\tau^{-1}=-2 \operatorname{Im} \Sigma_{i i}\left(\varepsilon_{i}\right)$,

and after noting the reality of the matrix elements of the LDA potential one finds Eq. (15).

Within many-body perturbation theory [96], it is possible to obtain $\Sigma\left(\boldsymbol{r}, \boldsymbol{r}^{\prime}, \omega\right)$ as a series in the Coulomb interaction $v\left(\boldsymbol{r}-\boldsymbol{r}^{\prime}\right)$. Due to the long range of this interaction, such a perturbation series is expected to contain divergent terms. However, it has 
been known for a long time that when the polarization induced in the system by the added electron is taken into account the series is free of divergences. Thus, the perturbation series for the self-energy can be rewritten in terms of the so-called screened interaction $W\left(\boldsymbol{r}, \boldsymbol{r}^{\prime} ; \omega\right)$.

To lowest order in the screened interaction, the self-energy reads:

$$
\begin{aligned}
& \Sigma\left(\boldsymbol{r}, \boldsymbol{r}^{\prime}, \omega\right) \\
& \quad=\mathrm{i} \int \frac{\mathrm{d} \omega^{\prime}}{2 \pi} \mathrm{e}^{\mathrm{i} \eta \omega^{\prime}} \mathscr{G}\left(\boldsymbol{r}, \boldsymbol{r}^{\prime}, \omega+\omega^{\prime}\right) W\left(\boldsymbol{r}, \boldsymbol{r}^{\prime}, \omega\right),
\end{aligned}
$$

which is the so-called GW approximation. The screened interaction is given by Eq. (16), in terms of the exact time-ordered density-response function of interacting electrons, or, equivalently, by the following integral equation

$$
\begin{aligned}
W\left(\boldsymbol{r}, \boldsymbol{r}^{\prime}, \omega\right)= & v\left(\boldsymbol{r}-\boldsymbol{r}^{\prime}\right)+\int \mathrm{d} \boldsymbol{r}_{1} \int \mathrm{d} \boldsymbol{r}_{2} v\left(\boldsymbol{r}-\boldsymbol{r}_{1}\right) \\
& \times \Pi\left(\boldsymbol{r}_{1}, \boldsymbol{r}_{2}, \omega\right) W\left(\boldsymbol{r}_{2}, \boldsymbol{r}^{\prime}, \omega\right), \quad(\mathrm{B}
\end{aligned}
$$

where $\Pi\left(\boldsymbol{r}, \boldsymbol{r}^{\prime}, \omega\right)$ represents the polarization propagator. In the GW approximation,

$$
\begin{aligned}
& \Pi^{G W}\left(\boldsymbol{r}, \boldsymbol{r}^{\prime}, \omega\right) \\
& =-\mathrm{i} \int \frac{\mathrm{d} \omega^{\prime}}{2 \pi} \mathscr{G}\left(\boldsymbol{r}, \boldsymbol{r}^{\prime}, \omega^{\prime}\right) \mathscr{G}\left(\boldsymbol{r}^{\prime}, \boldsymbol{r}, \omega^{\prime}-\omega\right) .
\end{aligned}
$$

If $\mathscr{G}$ is replaced in this expression by $\mathscr{G}^{0}\left(\Pi^{G W}\right) \rightarrow$ $\Pi^{0}$ ), one easily finds

$\operatorname{Re} \Pi^{0}\left(\boldsymbol{r}, \boldsymbol{r}^{\prime} ; \omega\right)=\operatorname{Re} \chi^{0}\left(\boldsymbol{r}, \boldsymbol{r}^{\prime} ; \omega\right)$

and

$\operatorname{Im} \Pi^{0}\left(\boldsymbol{r}, \boldsymbol{r}^{\prime} ; \omega\right)=\operatorname{sgn} \omega \operatorname{Im} \chi^{0}\left(\boldsymbol{r}, \boldsymbol{r}^{\prime} ; \omega\right)$,

where $\chi^{0}\left(\boldsymbol{r}, \boldsymbol{r}^{\prime} ; \omega\right)$ represents the retarded density-response function of noninteracting electrons, as defined by Eq. (A.7). For positive frequencies $(\omega>0)$, both $\Pi^{0}\left(\boldsymbol{r}, \boldsymbol{r}^{\prime} ; \omega\right)$ and $\chi^{0}\left(\boldsymbol{r}, \boldsymbol{r}^{\prime} ; \omega\right)$ coincide.

The GW approximation gives a comparatively simple expression for the self-energy operator, which allows the Green function of an interacting manyelectron system to be computed by simply starting from the Green function $\mathscr{G}^{0}\left(\boldsymbol{r}, \boldsymbol{r}^{\prime} ; \omega\right)$ of a fictitious system with an effective one-electron potential. The
GW approximation has been shown to be physically well motivated, especially for metals where the Hartree-Fock approximation leads to unphysical results.

Eqs. (B.3)-(B.11) form a set of equations which must be solved self-consistently for $\mathscr{G}\left(\boldsymbol{r}, \boldsymbol{r}^{\prime}, \omega\right)$. This means that the Green function used to calculate the self-energy must be found to coincide with the Green function obtained from the Dyson equation with the very same self-energy. However, there is some evidence [101] supporting the idea that introducing the noninteracting Green function $\mathscr{G}^{0}\left(\boldsymbol{r}, \boldsymbol{r}^{\prime} ; \omega\right)$ both in Eq. (B.9) and Eq. (B.11) $\left(G^{0} W^{0}\right.$ approximation) one obtains accurate results for the description of oneelectron properties such as the excitation energy and the quasiparticle lifetime. However, self-consistency modifies the one-electron excitation spectrum (excitation energies and lifetimes) [171,172], as well as the calculated screening properties. Self-consistent calculations have been performed only very recently for the homogeneous electron gas [171], simple semiconductors, and metals [172].

Discrepancies between $G^{0} W^{0}$ and self-consistent GW calculations seem to be originated in the fact that the so-called vertex corrections [100,101], which go beyond the GW approximation, need to be included as well. Inclusion of these corrections might cancel out the effect of self-consistency, thereby full self-consistent self-energy calculations yielding results that would be close to $G^{0} W^{0}$ results. The main outcome of self-consistent $\mathrm{GW}$ calculations for the electron gas is that the total energy [208] turns out to be strikingly close to the total energy calculated with use of quantum Monte Carlo techniques [198]. This result may be related to the fact that the self-consistent GW scheme conserves electron-number, energy, and total momentum, that is, fulfills the microscopic conservation laws [209].

The simplest improvement to the GW approximation is achieved by introducing a vertex correction that is consistent with an LDA calculation of the one-electron orbitals [210], the XC potential being regarded as a self-energy correction to the Hartree approximation. Based on this idea, the vertex $I$ [211] can be easily expressed in terms of the static local field correction of Eq. (A.9). This is the socalled GW $\Gamma$ approximation [102-105]. In this approximation, the polarization propagator is formally 
equivalent to the density-response function obtained within linear response theory in the framework of time-dependent density-functional theory [187-190]. In the case of a homogeneous electron gas, this approximation yields the test-charge-electron dielectric function of Eq. (A.23).

\section{Appendix C. Lifetimes of hot electrons near the Fermi level: Approximations}

The damping rate of hot electrons near the Fermi level $\left(E \ll E-E_{\mathrm{F}}\right)$ is obtained, within RPA, from Eq. (11) with the inverse dielectric function of Eq. (35), i.e.:

$$
\tau^{-1}=\frac{2}{\pi} \int_{0}^{2 q_{\mathrm{F}}} \frac{\mathrm{d} q}{q^{4}}\left|\epsilon_{q, 0}\right|^{-2} \frac{\left(E-E_{\mathrm{F}}\right)^{2}}{k_{i}} .
$$

For small values of $q\left(q \ll 2 q_{\mathrm{F}}\right)$,

$\epsilon_{q, 0} \approx\left(1-\beta \alpha^{2}\right)+\frac{\alpha^{2}}{z^{2}}$,

with

$$
\alpha=\frac{1}{\sqrt{\pi q_{\mathrm{F}}}},
$$

$z=q / 2 q_{\mathrm{F}}$, and $\beta=1 / 3$, and one obtains

$$
\begin{aligned}
\tau^{-1}= & \frac{\sqrt{\pi} q_{\mathrm{F}}^{-3 / 2}}{8 \sqrt{1-\beta \alpha^{2}}}\left[\arctan \frac{1}{\alpha_{0}}+\frac{\alpha_{0}}{1+\alpha_{0}^{2}}\right] \\
& \times \frac{\left(E-E_{\mathrm{F}}\right)^{2}}{k_{i}}
\end{aligned}
$$

where

$\alpha_{0}=\frac{\alpha}{\sqrt{1-\beta \alpha^{2}}}$.

This is the expression first obtained by Ritchie [39] and by Ritchie and Ashley [40].

In the high-density limit $\left(q_{\mathrm{F}} \rightarrow \infty\right)$, the static dielectric function of Eq. (C.2) is

$\epsilon_{q, 0}^{\mathrm{TF}} \approx 1+\frac{q_{\mathrm{TF}}^{2}}{q^{2}}$,

which can also be derived within the Thomas-Fermi statistical model, $q_{\mathrm{TF}}=\sqrt{4 q_{\mathrm{F}} / \pi}$ being the ThomasFermi momentum. By introducing Eq. (C.6) into Eq. (C.1) one obtains the expression derived by Quinn
[41], which can also be obtained by just taking $\beta=0$ in Eq. (C.4):

$$
\tau^{-1}=\frac{\sqrt{\pi} q_{\mathrm{F}}^{-3 / 2}}{8}\left[\arctan \frac{1}{\alpha}+\frac{\alpha}{1+\alpha^{2}}\right] \frac{\left(E-E_{\mathrm{F}}\right)^{2}}{k_{i}} .
$$

If one replaces the static dielectric function of Eq. (C.2) by the high-density limit $\left(q_{\mathrm{F}} \rightarrow \infty\right)$, as given by Eq. (C.6), and extends, at the same time, the integration of Eq. (C.1) to infinity, one finds Eq. (36), which can also be obtained by just keeping the first-order term in the expansion of Eq. (C.4) in $q_{\mathrm{F}}^{-1}$. If we further replace $k_{i} \rightarrow q_{\mathrm{F}}$ in Eq. (36), then the formula of Quinn and Ferrell is obtained [38]:

$\tau_{\mathrm{QF}}^{-1}=C\left(r_{s}\right)\left(E-E_{\mathrm{F}}\right)^{2}$,

with

$C\left(r_{s}\right)=\frac{\pi^{2} \sqrt{3}}{128} \frac{\omega_{p}}{E_{\mathrm{F}}^{2}}$,

or, equivalently,

$C\left(r_{s}\right)=\frac{\left(3 \pi^{2} / 2\right)^{1 / 3}}{36} r_{s}^{5 / 2}$.

In Fig. 18, the ratio $\tau / \tau_{\mathrm{QF}}$ is plotted against $r_{s}$ for hot electrons in the immediate vicinity of the

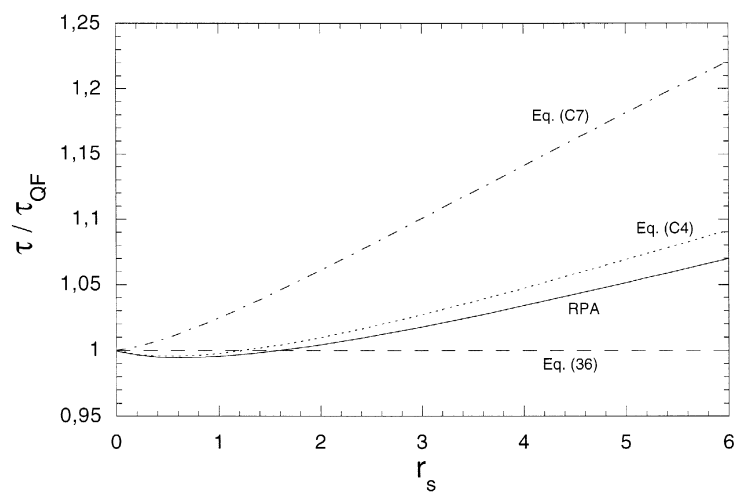

Fig. 18. Ratio $\tau / \tau_{\mathrm{QF}}$ between the lifetime $\tau$ evaluated in various approximations and the lifetime $\tau_{\mathrm{QF}}$ of Eq. (37), versus the electron-density parameter $r_{s}$, as obtained for hot electrons in the vicinity of the Fermi surface $\left(E \sim E_{\mathrm{F}}\right)$. The solid line represents the result obtained, within RPA, from either Eq. (11) or Eq. (C.1). Results obtained from Eqs. (C.4) and (C.7) are represented by dotted and dashed-dotted lines, respectively. The dashed line represents the result obtained from Eq. (36). 
Fermi surface $\left(k_{i} \sim q_{\mathrm{F}}\right)$, as computed from either Eq. (11) or Eq. (C.1) (solid line), and also from Eq. (C.4) (dotted line) and Eq. (C.7) (dashed-dotted line). In the range $0<r_{s}<6$, Eq. (C.4) reproduces the full RPA calculation within a $2 \%$, while either Eq. (36) or Eq. (C.8) reproduce the full calculation within a 7\%. Differences between the approximation of Quinn [41] (Eq. (C.7)) and the full RPA calculation go up to $14 \%$ at $r_{s}=6$.

Departures of the predictions of Eq. (C.4) (dotted line) from the full RPA calculation (solid line) arise from small differences between the dielectric function of Eq. (C.2) and the actual static RPA dielectric function. Further replacing the dielectric function of Eq. (C.2) by the high-density limit $\left(q_{\mathrm{F}} \rightarrow \infty\right)$, as given by Eq. (C.6), leads to a too-strong ThomasFermi-like screening and results, therefore, in a large overestimation of the lifetime (dashed-dotted line). However, this overestimation is largely compensated if one also takes $q_{\mathrm{F}} \rightarrow \infty$ in the integration of Eq. (C.1), thereby the lifetime of Quinn and Ferrell $\tau_{\mathrm{QF}}$ (dashed line) being closer to the full RPA calculation than the lifetime derived from Eq. (C.7) (dasheddotted line).

\section{References}

[1] R.H. Ritchie, F.W. Garber, M.Y. Nakai, R.D. Birkhoff, in Advances in Radiation Biology, vol. 3, p. 1, L.G. Augenstein, R. Mason, M. Zelle (Eds.), Academic Press, New York, 1969.

[2] R.H. Ritchie, C.J. Tung, V.E. Anderson, J.C. Ashley, Radiat. Res. 64 (1975) 181.

[3] H. Petek, S. Ogawa, Prog. Surf. Sci. 56 (1998) 239.

[4] H. Kanter, Phys. Rev. B 1 (1970) 522.

[5] C.J. Powell, Surf. Sci. 44 (1974) 29; Scanning Electron. Microsc. 184 (1984) 1649; Surf. Interface Anal. 7 (1985) 263.

[6] L.I. Johansson, B.E. Sernelius, Phys. Rev. B 50 (1994) 16817.

[7] J.B. Pendry, in Photoemission and the Electronic Properties of Surfaces, B. Feuerbacher, B. Fitton, R.F. Willis (Eds.), Wiley, 1978.

[8] W. Eberhardt, E.W. Plummer, Phys. Rev. B 21 (1980) 3245.

[9] H.J. Levinson, F. Greuter, E.W. Plummer, Phys. Rev. B 27 (1983) 727.

[10] A. Goldmann, W. Altmann, V. Dose, Solid State Commun. 79 (1991) 511.

[11] A. Santoni, F.J. Himpsel, Phys. Rev. B 43 (1991) 1305.
[12] D. Li, P.A. Dowben, J.E. Ortega, F.J. Himpsel, Phys. Rev. B 47 (1993) 12895.

[13] J. Bokor, Science 246 (1989) 1130.

[14] R. Haight, Surf. Sci. Rep. 21 (1995) 275.

[15] C.A. Schmutenmaer, M. Aeschlimann, H.E. Elsayed-Ali, R.J.D. Miller, D.A. Mantell, J. Cao, Y. Gao, Phys. Rev. B 50 (1994) 8957.

[16] E. Knoesel, A. Hotzel, T. Hertel, M. Wolf, G. Ertl, Surf. Sci. 368 (1996) 76.

[17] M. Aeschlimann, M. Bauer, S. Pawlik, Chem. Phys. 205 (1996) 127.

[18] S. Ogawa, H. Nagano, H. Petek, Phys. Rev. B 55 (1997) 10869.

[19] J. Cao, Y. Gao, R.J.D. Miller, H.E. Elsayed-Ali, D.A. Mantell, Phys. Rev. B 56 (1997) 1099.

[20] E. Knoesel, A. Hotzel, M. Wolf, Phys. Rev. B 57 (1998) 12812.

[21] A. Goldmann, R. Matzdorf, F. Theilmann, Surf. Sci. 414 (1998) L932.

[22] J. Cao, Y. Gao, H.E. Elsayed-Ali, R.J.D. Miller, D.A. Mantell, Phys. Rev. B 58 (1998) 10948.

[23] K. Reuter, P.L. de Andrés, F.J. García-Vidal, F. Flores, U. Hohenester, P. Kocevar, Europhys. Lett. 45 (1999) 181.

[24] R. Ludeke, A. Bauer, Phys. Rev. Lett. 71 (1993) 1760.

[25] M. Bauer, S. Pawlik, M. Aeschlimann, Proc. SPIE 3272 (1998) 201.

[26] M. Aeschlimann, M. Bauer, S. Pawlik, W. Weber, R. Burgermeister, D. Oberli, H.C. Siegmann, Phys. Rev. Lett. 79 (1997) 5158.

[27] M. Aeschlimann, R. Burgermeister, S. Pawlik, M. Bauer, D. Oberli, W. Weber, J. Elec. Spec. Rel. Phen. 88-91 (1998) 179.

[28] W. Nessler, S. Ogawa, H. Nagano, H. Petek, J. Shimoyama, Y. Nakayama, K. Kishio, Phys. Rev. Lett. 81 (1998) 4480.

[29] T. Hertel, E. Knoesel, M. Wolf, G. Ertl, Phys. Rev. Lett. 76 (1996) 535.

[30] M. Wolf, E. Knoesel, T. Hertel, Phys. Rev. B 54 (1996) 5295; M. Wolf, Surf. Sci. 377-379 (1997) 343.

[31] E. Knoesel, A. Hotzel, M. Wolf, J. Electron. Spectrosc. Relat. Phenom. 88-91 (1998) 577.

[32] R.L. Lingle Jr., N.H. Ge, R.E. Jordan, J.D. McNeil, C.B. Harris, Chem. Phys. 205 (1996) 191.

[33] U. Höfer, I.L. Shumay, Ch. Reuss, U. Thomann, W. Wallauer, Th. Fauster, Science 277 (1997) 1480.

[34] J.D. McNeil, R.L. Lingle Jr., N.H. Ge, C.M. Wong, R.E. Jordan, C.B. Harris, Phys. Rev. Lett. 79 (1997) 4645; C.B. Harris, N.-H. Ge, R.L. Lingle Jr., J.D. McNeill, C.M. Wong, Annu. Rev. Phys. Chem. 48 (1997) 711.

[35] I.L. Shumay, U. Höfer, Ch. Reuss, U. Thomann, W. Wallauer, Th. Fauster, Phys. Rev. B 58 (1998) 13974.

[36] W. Plummer, Science 277 (1997) 1447.

[37] W.S. Fann, R. Storz, H.W.K. Tom, Phys. Rev. B 46 (1992) 13592.

[38] J.J. Quinn, R.A. Ferrell, Phys. Rev. 112 (1958) 812.

[39] R.H. Ritchie, Phys. Rev. 114 (1959) 644.

[40] R.H. Ritchie, J.C. Ashley, J. Phys. Chem. Solids 26 (1963) 1689. 
[41] J.J. Quinn, Phys. Rev. 126 (1962) 1453.

[42] S.L. Adler, Phys. Rev. 130 (1963) 1654.

[43] J.J. Quinn, Appl. Phys. Lett. 2 (1963) 167.

[44] L. Kleinman, Phys. Rev. B 3 (1971) 2982.

[45] D.R. Penn, Phys. Rev. B 13 (1976) 5248.

[46] C.A. Kukkonen, A.W. Overhauser, Phys. Rev. B 20 (1979) 550.

[47] D.R. Penn, Phys. Rev. B 22 (1980) 2677.

[48] B.I. Lundqvist, Phys. Stat. Sol. 32 (1969) 273.

[49] J.C. Shelton, Surf. Sci. 44 (1974) 305.

[50] J.C. Ashley, R.H. Ritchie, Phys. Status Solidi B 62 (1974) 253; 83 (1977) K159.

[51] J.C. Ashley, C.J. Tung, R.H. Ritchie, Surf. Sci. 81 (1979) 409.

[52] C.J. Tung, R.H. Ritchie, Phys. Rev. B 16 (1977) 4302.

[53] W.F. Krolikowski, W.E. Spicer, Phys. Rev. 185 (1969) 882.

[54] C.J. Tung, J.C. Ashley, R.H. Ritchie, Surf. Sci. 81 (1979) 427.

[55] J.C. Ashley, J. Electron Spectrosc. 28 (1982) 177; 46 (1988) 199; 50 (1990) 323; J. Phys-Condens. Mat. 3 (1991) 2741.

[56] J. Szajman, R.C.G. Leckey, J. Electron Spectrosc. 23 (1981) 83.

[57] D. Liljequist, J. Phys. D: Appl. Phys. 16 (1983) 1567.

[58] F. Salvat, J.D. Martínez, R. Mayol, J. Parellada, J. Phys. D: Appl. Phys. 18 (1985) 299; J.M. Fernández-Varea, R. Mayol, F. Salvat, D. Liljequist, J. Phys.: Condens. Matter 4 (1992) 2879.

[59] D.R. Penn, Phys. Rev. B 35 (1987) 482.

[60] S. Tanuma, C.J. Powell, D.R. Penn, Surf. Interface Anal. 11 (1988) 577; 17 (1991) 911; 17 (1991) 927; 20 (1993) 77; 21 (1994) 165.

[61] C.M. Kwei, Y.F. Chen, C.J. Tung, J.P. Wang, Surf. Sci. 293 (1993) 202; Z.-J. Ding, R. Shimizu, Scanning 18 (1996) 92; T. Boutboul, A. Akkerman, A. Breskin, R. Chechik, J. Appl. Phys. 79 (1996) 6714; A. Akkerman, T. Boutboul, A. Breskin, R. Chechik, A. Gibrekhterman, Y. Lifshitz, Phys. Stat. Sol. (b) 198 (1996) 769.

[62] C.J. Powell, A. Jablonski, J. Phys. Chem. Ref. Data 28 (1999) 19

[63] I. Campillo, PhD. Thesis, University of the Basque Country, 1999 (unpublished); I. Campillo, J.M. Pitarke, A. Rubio, P.M. Echenique, to be published.

[64] I. Campillo, J.M. Pitarke, A. Rubio, E. Zarate, P.M. Echenique, Phys. Rev. Lett. 83 (1999) 2230.

[65] P.M. Echenique, F. Flores, F. Sols, Phys. Rev. Lett. 55 (1985) 2348.

[66] P.L. de Andrés, P.M. Echenique, F. Flores, Phys. Rev. B 35 (1987) 4529; Phys. Rev. B 39 (1989) 10356.

[67] M.E. Uranga, A. Rivacoba, P.M. Echenique, Progr. Surf. Sci. 42 (1993) 67.

[68] J. Rundgren, G. Malmstrom, J. Phys. C 10 (1977) 4671.

[69] P.M. Echenique, PhD. Thesis, University of Cambridge, 1976 (unpublished); P.M. Echenique, J.B. Pendry, J. Phys. C 11 (1978) 2065.

[70] N.V. Smith, Phys. Rev. 32 (1985) 3549; N.V. Smith, Rep. Progr. Phys. 51 (1988) 1227.
[71] F.J. Himpsel, Comments Cond. Matter Phys. 12 (1986) 199.

[72] G. Borstel, G. Thörner, Surf. Sci. Rep. 8 (1988) 1.

[73] P.M. Echenique, J.B. Pendry, Prog. Surf. Sci. 32 (1990) 111.

[74] Th. Fauster, W. Steinmann, in Electromagnetic Waves: Recent Development in Research, vol. 2, p. 350, P. Halevi (Ed.), Elsevier, Amsterdam, 1995.

[75] R.M. Osgood Jr., X. Wang, Solid State Phys. 51 (1997) 1.

[76] S. Gao, B.I. Lundqvist, Prog. Theor. Phys. Suppl. 106 (1991) 405; Solid State Commun. 84 (1992) 147.

[77] E.V. Chulkov, I. Sarria, V.M. Silkin, J.M. Pitarke, P.M. Echenique, Phys. Rev. Lett. 80 (1998) 4947.

[78] J. Osma, I. Sarria, E.V. Chulkov, J.M. Pitarke, P.M. Echenique, Phys. Rev. B 59 (1999) 10591.

[79] E.V. Chulkov, J. Osma, I. Sarria, V.M. Silkin, J.M. Pitarke, Surf. Sci. 433-435 (1999) 882.

[80] I. Sarria, J. Osma, E.V. Chulkov, J.M. Pitarke, P.M. Echenique, Phys. Rev. B 60 (1999) 11795.

[81] E.V. Chulkov, V.M. Silkin, P.M. Echenique, Surf. Sci. 391 (1997) L1217.

[82] C.C. Sung, R.H. Ritchie, Phys. Rev. A 28 (1983) 674.

[83] C.D. Hu, E. Zaremba, Phys. Rev. B 37 (1988) 9268.

[84] H. Esbensen, P. Sigmund, Ann. Phys. (N.Y.) 201 (1990) 152.

[85] J.M. Pitarke, R.H. Ritchie, P.M. Echenique, E. Zaremba, Europhys. Lett. 24 (1993) 613; J.M. Pitarke, R.H. Ritchie, P.M. Echenique, Nucl. Instrum. Methods B 79 (1993) 209.

[86] J.M. Pitarke, R.H. Ritchie, P.M. Echenique, Phys. Rev. B 52 (1995) 13883.

[87] J.M. Pitarke, A. Bergara, R.H. Ritchie, Nucl. Instrum. Methods B 99 (1995) 87; A. Bergara, I. Campillo, J.M. Pitarke, P.M. Echenique, Phys. Rev. 56 (1997) 15654.

[88] N.-P. Wang, J.M. Pitarke, Phys. Rev. A 56 (1997) 2913; N.-P. Wang, J.M. Pitarke, Phys. Rev. A 57 (1998) 4053; N.-P. Wang, J.M. Pitarke, Nucl. Instrum. Methods B 135 (1998) 92

[89] W.H. Barkas, W. Birnbaum, F.M. Smith, Phys. Rev. 101 (1956) 778; W.H. Barkas, N.J. Dyer, H.H. Heckman, Phys. Rev. Lett. 11 (1963) 26; 11 (1963) 138E.

[90] L.H. Andersen, P. Hvelplund, H. Knudsen, S.P. Moller, J.O.P. Pedersen, E. Uggerhoj, K. Elsener, E. Morenzoni, Phys. Rev. Lett. 62 (1989) 1731.

[91] L.I. Shiff, Quantum Mechanics, McGraw-Hill, London, 1985; A. Galindo, P. Pascual, Quantum Mechanics, Springer-Verlag, Berlin, 1990.

[92] P.M. Echenique, F. Flores, R.H. Ritchie, Solid State Phys. 43 (1990) 229.

[93] D. Pines, P. Nozieres, The Theory of Quantum Liquids, vol. I: Normal Fermi Liquids, Addison-Wesley, New York, 1989

[94] J. Lindhard, K. Dan. Vidensk. Selsk. Mat.- Fys. Medd. 28, No. 8 (1954).

[95] D. Pines, Elementary excitations in solids, Addison-Wesley, New York, 1963

[96] A.L. Fetter, J.D. Walecka, Quantum Theory of Many-Particle Systems, McGraw-Hill, New York, 1971; A.A. Abrikosov, L.P. Gorkov, I.E. Dzyaloshinski, Methods of 
Quantum Field Theory in Statistical Physics, Dover Publications, New York, 1975.

[97] J. Hubbard, Proc. R. Soc. London, Ser. A 240 (1957) 539; 243 (1957) 336.

[98] L. Kleinman, Phys. Rev. 172 (1968) 383.

[99] L. Hedin, B.I. Lundqvist, J. Phys. C 4 (1971) 2064.

[100] L. Hedin, Phys. Rev. 139 (1965) A796; L. Hedin, S. Lundqvist, Solid State Phys. 23 (1969) 1.

[101] F. Aryasetiawan, O. Gunnarsson, Rep. Prog. Phys. 61 (1998) 237.

[102] T.M. Rice, Ann. Phys. (N.Y.) 31 (1965) 100.

[103] G.D. Mahan, B.E. Sernelius, Phys. Rev. Lett. 62 (1989) 2718.

[104] G.D. Mahan, Many-Particle Physics, 2nd ed., Plenum, New York, 1990.

[105] G.D. Mahan, Comments Cond. Mat. Phys. 16 (1994) 333.

[106] J. Lindhard, M. Scharff, K. Dan. Vidensk. Selsk. Mat.-Fys. Medd 27, No. 15 (1953); J. Lindhard, M. Scharff, H.E. Schiott, ibid. 33, No. 14 (1963).

[107] N.W. Ashcroft, N.D. Mermin, Solid State Physics, Saunders, Philadelphia, 1976.

[108] E.D. Palik, Handbook of Optical Constants of Solids, Academic Press, New York, 1985; E.D. Palik, Handbook of Optical Constants of Solids II, Academic Press, New York, 1991.

[109] E. Zarate, PhD. Thesis, University of the Basque Country, 1999 (unpublished); E. Zarate, P. Apell, P.M. Echenique, Phys. Rev. B 60 (1999) 2326.

[110] C.N. Berglund, W.E. Spicer, Phys. Rev. 136 (1964) 1030.

[111] E.O. Kane, Phys. Rev. 159 (1967) 624.

[112] D.R. Penn, S.P. Apell, S.M. Girvin, Phys. Rev. Lett. 55 (1985) 518; ibid. Phys. Rev. B 32 (1985) 7753.

[113] H.-J. Drouhin, Phys. Rev. B 56 (1997) 14886.

[114] P. Hohenberg, W. Kohn, Phys. Rev. 136 (1964) B864.

[115] W. Kohn, L. Sham, Phys. Rev. 140 (1965) A1133.

[116] N. Troullier, J.L. Martins, Phys. Rev. B 43 (1991) 1993.

[117] I.E. Tamm, Z. Phys. 76 (1932) 849.

[118] W. Shockley, Phys. Rev. 56 (1939) 317.

[119] P.D. Johnson, N.V. Smith, Phys. Rev. B 27 (1983) 2527.

[120] V. Dose, W. Altmann, A. Goldmann, U. Kolac, J. Rogozik, Phys. Rev. Lett. 52 (1984) 1919.

[121] D. Straub, F.J. Himpsel, Phys. Rev. Lett. 52 (1984) 1922.

[122] F.J. Himpsel, J.E. Ortega, Phys. Rev. B 46 (1992) 9719.

[123] K. Giesen, F. Hage, F.J. Himpsel, H.J. Riess, W. Steinmann, Phys. Rev. Lett. 55 (1985) 300.

[124] R.W. Schoenlein, J.G. Fujimoto, G.L. Eesley, T.W. Capehart, Phys. Rev. Lett. 61 (1988) 2596; R.W. Schoenlein, J.G. Fujimoto, G.L. Eesley, T.W. Capehart, Phys. Rev. B 43 (1991) 4688.

[125] S. Schuppler, N. Fischer, Th. Fauster, W. Steinmann, Phys. Rev. B 46 (1992) 13539.

[126] W. Wallauer, Th. Fauster, Surf. Sci. 374 (1997) 44.

[127] M. Weinert, S.L. Hulbert, P.D. Johnson, Phys. Rev. Lett. 55 (1985) 2055.

[128] M. Ortuño, P.M. Echenique, Phys. Rev. B 34 (1986) 5199.
[129] J.B. Pendry, C.G. Larsson, P.M. Echenique, Surf. Sci. 166 (1986) 57.

[130] Z. Lenac, M. Šunjić, H. Conrad, M.E. Kordesch, Phys. Rev. B 36 (1987) 9500.

[131] S. Å. Lindgren, L. Walldén, Phys. Rev. B 40 (1989) 11546.

[132] N.V. Smith, C.T. Chen, M. Weinert, Phys. Rev. B 40 (1989) 7565.

[133] Th. Fauster, Appl. Phys. A 59 (1994) 639.

[134] L. Jurczyszyn, M. Stȩślicka, Surf. Sci. 376 (1997) L424.

[135] E.V. Chulkov, V.M. Silkin, P.M. Echenique, Surf. Sc. 437 (1999) 30

[136] S.L. Hulbert, P.D. Johnson, M. Weinert, R.F. Garrett, Phys. Rev. B 33 (1986) 760.

[137] M. Nekovee, J.E. Inglesfield, Europhys. Lett. 19 (1992) 535.

[138] A.G. Eguiluz, M. Heinrichsmeier, A. Fleszar, W. Hanke, Phys. Rev. Lett. 68 (1992) 1359.

[139] M. Nekovee, S. Crampin, J.E. Inglesfield, Phys. Rev. Lett. 70 (1993) 3099.

[140] V.M. Silkin, E.V. Chulkov, Phys. Solid State 36 (1994) 404 [Rus. Fiz. Tverd. Tela 36 (1994) 736].

[141] Z. Li, S. Gao, Phys. Rev. B 50 (1994) 15349.

[142] M. Heinrichsmeier, A. Fleszar, W. Hanke, A. Eguiluz, Phys. Rev. B 57 (1998) 14974.

[143] S.D. Kevan (Ed.), Angle-Resolved Photoemission, Theory and Current Applications, Elsevier, Amsterdam, 1992.

[144] M. Weinelt, Ch. Reuss, M. Kutschera, U. Thomann, I.L. Shumay, Th. Fauster, U. Höfer, F. Theilmann, A. Goldmann, to appear in Appl. Phys. B. - Lasers, 68 (1999) 377.

[145] B.A. McDougall, T. Balasubramanian, E. Jensen, Phys. Rev. B 51 (1995) 13891.

[146] F. Theilmann, R. Matzdorf, G. Meister, A. Goldmann, Phys. Rev. B 56 (1997) 3632.

[147] Ch. Reuss, I.L. Shumay, V. Thomann, M. Kutschera, M. Weinelt, Th. Fauster, U. Höfer, Phys. Rev. Lett. 82 (1999) 153.

[148] E.V. Chulkov, V.M. Silkin, P.M. Echenique, to be published.

[149] J.J. Deisz, A.G. Eguiluz, Phys. Rev. B 55 (1997) 9195.

[150] D.F. Padowitz, W.R. Merry, R.E. Jordan, C.B. Harris, Phys. Rev. Lett. 69 (1992) 3583.

[151] N. Fischer, S. Schuppler, Th. Fauster, W. Steinmann, Surf. Sci. 314 (1994) 89.

[152] N. Fischer, S. Schuppler, R. Fischer, Th. Fauster, W. Steinmann, Phys. Rev. B 47 (1993) 4705.

[153] R. Fischer, Th. Fauster, Surf. Rev. Lett. 3 (1996) 1783.

[154] W. Merry, R.E. Jordan, D.E. Padowitz, C.B. Harris, Surf. Sci. 295 (1993) 393.

[155] J.D. McNeill, R.L. Lingle Jr., R.E. Jordan, D.F. Padowitz, C.B. Harris, J. Chem. Phys. 105 (1996) 3883.

[156] M. Wolf, Surf. Sci. 377-379 (1997) 343.

[157] C.B. Harris, N.H. Ge, R.L. Lingle Jr., J.D. McNeill, C.M. Wong, Annu. Rev. Phys. Chem. 48 (1997) 711.

[158] W. Berthold, I.L. Shumay, P. Feulner, U. Hofer, to be published. 
[159] M. Donath, Surf. Sci. Rep. 20 (1994) 251.

[160] F. Passek, M. Donath, K. Ertl, V. Dose, Phys. Rev. Lett. 75 (1995) 2746.

[161] M. Nekovee, S. Crampin, J.E. Inglesfield, Phys. Rev. Lett. 70 (1993) 3099.

[162] S. De Rossi, F. Cicacci, S. Crampin, Phys. Rev. Lett. 77 (1996) 908.

[163] J. Osma (unpublished).

[164] E.W.G. Diau, J.L. Herek, Z.H. Kim, A.H. Zewail, Science 279 (1998) 847.

[165] L. Petaccia, L. Grill, M. Zangrando, S. Modesti, Phys. Rev. Lett. 82 (1999) 386.

[166] T. Reiners, H. Haberland, Phys. Rev. Lett. 77 (1996) 2440.

[167] A. Domps, P.G. Reinhard, E. Suraud, Phys. Rev. Lett. 81 (1998) 5524.

[168] B.L. Altshuler, Y. Gefen, A. Kamenev, L.S. Levitov, Phys. Rev. Lett. 78 (1997) 2803.

[169] M. Rohfling, S.G. Louie, Phys. Rev. Lett. 81 (1998) 2312; L.X. Benedict, E.L. Shirley, R.B. Bohn, Phys. Rev. Lett. 80 (1998) 4514; S. Albretcht, L. Reining, R. Del Sole, G. Onida, Phys. Rev. Lett. 80 (1998) 4510.

[170] E. Shirley, Phys. Rev. B 54 (1996) 7758; 54 (1996) 8411.

[171] B. Holm, U. Von Barth, Phys. Rev. B 57 (1998) 2108.

[172] W.-D. Schöne, A.G. Eguiluz, Phys. Rev. Lett. 81 (1998) 1662; A.G. Eguiluz, W.-D. Schöne, Mol. Phys. 94 (1998) 87.

[173] F. Aryasetiawan, Phys. Rev. B 46 (1992) 13051.

[174] W. Gerhardt, S. Marquardt, N. Schroeder, S. Weis, Phys. Rev. B 58 (1998) 6877.

[175] M.S. Hybertsen, S.G. Louie, Phys. Rev. Lett. 55 (1985) 1418; M.S. Hybertsen, S.G. Louie, Phys. Rev. B 34 (1986) 5390; R.W. Godby, M. Schlüter, L.J. Sham, Phys. Rev. Lett. 56 (1986) 2415; Phys. Rev. B 37 (1988) 10159.

[176] J.S. Dolado, M.A. Cazalilla, A. Rubio, P.M. Echenique (unplublished).

[177] H.N. Rojas, R.W. Godby, R.J. Needs, Phys. Rev. Lett. 74 (1995) 1827.

[178] X. Blase, A. Rubio, M.L. Cohen, S.G. Louie, Phys. Rev. B 52 (1995) 2225.

[179] X. Blase, A. Rubio, M.L. Cohen, S.G. Louie (unpublished).

[180] L. Steinbeck, A. Rubio, I.D. White, R.W. Godby, to be published.

[181] S. Xu, J. Cao, CC. Miller, D.A. Mantell, R.J.D. Miller, Y. Gao, Phys. Rev. Lett. 76 (1996) 483.

[182] L. Zheng, S. Das Sarma, Phys. Rev. Lett. 77 (1996) 1410; S. Xu, J. Cao, CC. Miller, D.A. Mantell, R.J.D. Miller, Y. Gao, Phys. Rev. Lett. 77 (1996) 1411.

[183] J. Gonzalez, F. Guinea, M.A.H. Vozmediano, Phys. Rev. Lett. 77 (1996) 3589

[184] S. Xu, CC. Miller, S.J. Diol, Y. Gao, D.A. Mantell, M.G. Mason, A.A. Muenter, L.I. Sharp, B.A. Parkinson, R.J.D. Miller, Chem. Phys. Lett. 272 (1997) 209.
[185] M.A. Cazalilla, A. Rubio, P.M. Echenique (unpublished).

[186] P. Laitenberger, R.E. Palmer, Phys. Rev. Lett. 76 (1996) 1952.

[187] E. Runge, E.K.U. Gross, Phys. Rev. Lett. 52 (1984) 997.

[188] E.K.U. Gross, W. Kohn, Phys. Rev. Lett. 55 (1985) 2850; 57 (1986) 923E.

[189] M. Petersilka, U.J. Gossmann, E.K.U. Gross, Phys. Rev. Lett. 76 (1996) 1212.

[190] E.K.U. Gross, F.J. Dobson, M. Petersilka, Density Functional Theory, Springer, New York, 1996.

[191] H. Flocard, S.E. Koonin, M.S. Weiss, Phys. Rev. C 17 (1978) 1682.

[192] A. Rubio, J.A. Alonso, X. Blase, S.G. Louie, Int. J. Mod. Phys. B 11 (1997) 2727, and references therein.

[193] K. Yabana, G.F. Bertsch, Phys. Rev. B 54 (1996) 4484.

[194] A. Rubio, G.F. Berstch (unpublished).

[195] E.P. Wigner, Phys. Rev. 46 (1934) 1002; Trans. Faraday Soc. 34 (1938) 678.

[196] S.H. Vosko, L. Wilk, M. Nusair, Can. J. Phys. 58 (1980) 1200.

[197] J. Perdew, A. Zunger, Phys. Rev. B 23 (1981) 5048.

[198] D.M. Ceperley, B.J. Alder, Phys. Rev. Lett. 45 (1980) 1196.

[199] C. Bowen, G. Sugiyama, B.J. Alder, Phys. Rev. 50 (1994) 14838 .

[200] S. Moroni, D.M. Ceperley, G. Senatore, Phys. Rev. Lett. 75 (1995) 689.

[201] K.S. Singwi, M.P. Tosi, R.H. Land, A. Sjolander, Phys. Rev. 176 (1968) 589; K.S. Singwi, A. Sjolander, M.P. Tosi, R.H. Land, ibid. 1 (1970) 1044; K.S. Singwi, M.P. Tosi, Solid State Phys. 36 (1981) 177.

[202] K. Utsumi, S. Ichimaru, Phys. Rev. B 22 (1980) 5203; S. Ichimaru, Rev. Mod. Phys. 54 (1982) 1017.

[203] A. Gold, L. Camels, Phys. Rev. B 48 (1993) 11622.

[204] F. Brosens, L.F. Lemmens, J.T. Devreese, Phys. Status Solid B 74 (1976) 45; J.T. Devreese, F. Brosens, L.F. Lemmens, Phys. Rev. B 21 (1980) 1349.

[205] C.F. Richardson, N.W. Ashcroft, Phys. Rev. B 50 (1994) 8170.

[206] B.C. Larson, J.Z. Tischler, E.D. Isaacs, P. Zschack, A. Fleszar, A.G. Eguiluz, Phys. Rev. Lett. 77 (1996) 1346.

[207] P. Nozieres, Theory of Interacting Fermi Liquids, Benjamin, New York, 1962.

[208] V.M. Galitski, A.B. Migdal, Sov. Phys. JEPT 7 (1958) 96.

[209] G. Baym, L.P. Kadanoff, Phys. Rev. 124 (1961) 287; L.P. Kadanoff, G. Baym, Quantum Statistical Mechanics, Benjamin, New York, 1962.

[210] R.M. Dreizler, E.K.U. Gross, Density Functional Theory, an Approach to the Quantum Many Body Problem, Springer, Berlin, 1990

[211] R. Del Sole, L. Reining, R.W. Godby, Phys. Rev. B 49 (1994) 8024. 